\title{
On the shock-induced variability of emission lines in M-type Mira variables
}

\section{Observational data}

\author{
He. Richter ${ }^{1, \star}$ and P. R. Wood ${ }^{2}$ \\ 1 Institut für Astronomie und Astrophysik, TU Berlin, Sekr. PN 8-1, Hardenbergstraße 36, 10623 Berlin, Germany \\ 2 Research School of Astronomy and Astrophysics, Australian National University, Cotter Road, \\ Weston ACT 2611, Australia \\ e-mail: wood@mso.anu.edu.au
}

Received 30 October 2000 / Accepted 25 January 2001

\begin{abstract}
We present time-resolved observations of metallic emission lines of MgI, Mn I, SiI, Fe I and FeII, including forbidden emission lines of [Fe II], for the six M-type Mira variables RR Sco, R Aql, R Car, R Leo, S Scl and R Hya, which range in period from 281 to 389 days. Data is also presented for the Balmer emission lines $\mathrm{H} \gamma, \mathrm{H} \delta, \mathrm{H} \zeta$ and $\mathrm{H} \eta$. The observations were carried out in the optical wavelength region 3600-5700 A. Narrow-slit observations with dispersion of $1.53 \mathrm{~km} \mathrm{~s}^{-1}$ pixel $^{-1}$ and a 3-pixel resolution of $4.6 \mathrm{~km} \mathrm{~s}^{-1}$ were made in some cases while most observations were done with a wide slit corresponding to a resolution of $15.3 \mathrm{~km} \mathrm{~s}^{-1}$. The variation of line shape, flux and velocity with phase is discussed. The data presented in this paper will be used in subsequent papers for comparison with detailed models of the emission from shock waves in the upper atmospheric layers of Mira variables. It is in these important upper layers that dust formation occurs and mass loss is initiated.
\end{abstract}

Key words. line: profiles - shock waves - stars: circumstellar matter - stars: late-type - stars: variables: general

\section{Introduction}

Two of the outstanding problems concerning M-type Miras are the basic mechanism for dust formation and the mechanism for the generation of high mass loss rates of the order of $10^{-6} M_{\odot} \mathrm{yr}^{-1}$ (Habing 1996). Theoretical works of Wood (1979), Bowen (1988), Fleischer et al. (1992), Feuchtinger et al. (1993), Höfner \& Dorfi (1997) and Winters et al. (2000) show that the high mass loss rates of Miras can be explained by a combination of pulsation of the star and radiation pressure acting on dust. Pulsation generates shock waves which move out through the atmosphere thereby increasing the mass density in the outer parts of the atmosphere. The passage of a shock leads to a sudden increase of the pressure, density and temperature of the gas. Behind the shock the gas cools via the emission of radiation, which leads to conditions that are favourable for dust formation. The shocks in the outer parts of the atmosphere thereby trigger the formation of

Send offprint requests to: He. Richter,

e-mail: richter@astro.physik.tu-berlin.de

* Visitor (May-November 1999) in the Research School of Astronomy and Astrophysics, Australian National University. dust. Radiation pressure acting on this dust leads to the high mass loss rate.

One way to reveal the thermo- and hydro-dynamical conditions in a Mira atmospheres is to study the various emission lines which are emitted behind the shock front and which can be observed over a substantial portion of the pulsation period. Analysing a time-resolved series of these emission lines offers the possibility to determine the hydrodynamical conditions in different layers of the atmosphere influenced by the passing shock wave. In particular, the hydrodynamical conditions of the outer, dustproducing layers of the atmosphere can be studied with the help of metal emission lines which appear late in the pulsation cycle when the shock wave has reached these layers.

M-type Miras show a variety of metal emission lines in all regions of wavelength. The most substantial observations in the optical wavelength region are the long-term observation of $o$ Ceti by Joy (1954) and the observation of several Miras by Merrill (1940, 1945, 1946a, 1946b, 1947a, $1947 \mathrm{~b})$. In the near-IR wavelength region $(\lambda<1.01 \mu \mathrm{m})$, Gillet et al. 1985a, 1985b, 1985c) observed various metal emission lines of SCar and $o$ Ceti and interpreted the variation of the lines using a shock model. Using ISO, 
Aoki et al. (1998) found a number of high intensity emission lines of ionized metals in the mid-IR spectral region, although the stars examined were semi-regular and irregular variables rather than Miras. Finally, in the UV spectral region, Wood \& Karovska (2000) observed emission lines in M-type Miras and suggested shock waves as an explanation for their variations.

Around maximum light, the hydrogen Balmer emission lines are prominent in the optical spectra. Deutsch \& Merrill (1959) were the first to interpret these lines as due to shock waves and the theoretical work of Gorbatski (1961) verified their interpretation. A number of observational studies of Balmer emission lines have been made over the years, many concentrating on the mean radial velocity (Merrill 1940, 1945, 1946a, 1946b, 1947a, 1947b; Joy 1947, 1954; Gillet et al. 1985a, 1985b, 1985c). Quantitive data on Balmer line shapes, widths and line fluxes, as well as theoretical shock model estimates for the shock speed and temperature and line fluxes, are presented in Fox et al. (1984, 1985) and Gillet et al. (1983, 1985c).

The emphasis of our work is on various metal emission lines of Fe I, Fe II, MgI, Mn I and Si I, especially the lines which appear at late phases and which therefore should originate from the outer parts of the atmosphere. For two of the stars in our sample (R Car and R Leo) we observed forbidden lines [Fe II] around minimum light. These lines were observed by Joy (1954) in o Ceti as well as by Merrill (1940, 1947b) in the M-type Miras U Ori, R Leo, R Hya and the S-type Mira $\chi$ Cyg. These lines must occur only very briefly in each cycle or perhaps only in occasional cycles as we did not see the lines in R Leo during the minimum of March 1999 whereas the lines were strong during the minimum of December 1999. Similarly, we did not see the lines during the minima of $o$ Ceti (which we also observed, but do not present in this paper since no metal emission lines were detected) or R Hya although the lines have been reported in the past in these stars. We also note that although Joy (1954) reports the lines in o Ceti, Merrill (1940) explicitly notes that he did not see them.

Existing spectral observations have mostly concentrated on the mean radial velocities of the metal emission lines. There is very little published quantitive data on the shapes, widths and fluxes of metal emission lines. Here we present these quantities for a sample of six M-type Miras, namely R Aql, RR Sco, R Car, R Leo, S Scl and RHya, which range in period from 281 days to 389 days. These Miras have been observed at multiple phases of the pulsation cycle. Because of this phase coverage, the data shows the history of the shock as it emerges through the deep photosphere (before maximum light) and then moves out through the atmosphere.

As well as the metal line observations, we also present similar data for the hydrogen emission lines $\mathrm{H} \gamma, \mathrm{H} \delta, \mathrm{H} \zeta$, $\mathrm{H} \eta$. These lines are most prominent around maximum light and they provide information about early history of the specific shock wave that generated the metal lines we observed.
Table 1. The M-type Mira Sample

\begin{tabular}{lclr}
\hline Star & $P[$ days $]$ & Sp. & $V_{*}[\mathrm{~km} / \mathrm{s}]$ \\
\hline RR Sco & 281 & M6e-M9e & $-37^{a}$ \\
R Aql & 284 & M5e-M9e & $29^{b}$ \\
R Car & 309 & M4e-M8e & $20^{c}$ \\
R Leo & 310 & M6e-M9.5e & $6^{b}$ \\
S Scl & 363 & M3e-M9e(Tc) & $(12)$ \\
R Hya & 389 & M6e-M9eS(Tc) & $-11^{b}$ \\
\hline
\end{tabular}

${ }^{\mathrm{a}} V_{*}$ from the midpoint of the CO $J=3-2$ transition (Young 1995).

b $V_{*}$ from the midpoint of the $\mathrm{CO} J=2-1$ transition (Cernicharo et al. 1997).

c $V_{*}$ from the midpoint of the $\mathrm{SiO} J=3-2, v=0$ transition (Groenewegen et al. 1999).

\section{Observations and data reduction}

The observations presented here were taken from December 1998 to December 1999. The observed line spectra were obtained with the coudé echelle spectrograph and $81 \mathrm{~cm}$ camera of the Mount Stromlo Observatory $1.88 \mathrm{~m}$ telescope. A Site $2 \times 4 \mathrm{k}$ CCD was used as detector, allowing coverage of the spectral region $3600-5700 \AA$ in each exposure: the corresponding spectral range in each echelle order was 70-60 . The echelle grating gave a dispersion of $0.0204 \AA$ pixel $^{-1}$ at $4000 \AA$, equivalent to $1.53 \mathrm{~km} \mathrm{~s}^{-1}$ pixel $^{-1}$. Maximum resolution observations were done with a $300 \mu \mathrm{m}\left(1.2^{\prime \prime}\right)$ slit, giving a 3-pixel resolution of $4.6 \mathrm{~km} \mathrm{~s}^{-1}$. For spectophotometric observations, a $1500 \mu \mathrm{m}\left(6^{\prime \prime}\right)$ slit was used, giving a resolution of $15.3 \mathrm{~km} \mathrm{~s}^{-1}$. Because of the high slit losses experienced with the $300 \mu \mathrm{m}$ slit, and the fact that the emission lines are mostly considerably broader than $15.3 \mathrm{~km} \mathrm{~s}^{-1}$, a large fraction of all observations were in fact done using the wide slit.

The periods, spectral types and stellar center-of-mass velocities of the Miras in our sample are listed in Table 1. The periods and spectral types are taken from the index to variable stars of the Variable Star Network (VSNET) ${ }^{1}$ (Nogami et al. 1997). The heliocentric stellar center-ofmass velocity $v_{*}$ was determined from the midpoint of the circumstellar molecular emission of $\mathrm{CO}$ or $\mathrm{SiO}$ given in the papers cited below the table. This method of deriving stellar center-of-mass velocities has already been described by Reid \& Dickinson (1976) and the velocities should be correct to $1-2 \mathrm{~km} \mathrm{~s}^{-1}$. For S Scl it was not possible to find a direct estimate of $v_{*}$ in the literature. We adopted the value given in parentheses: it was obtained by choosing $v_{*}$ to give the same velocity (relative to the stellar centerof-mass) for various emission lines as found for stars with known $v_{*}$. This estimate is probably correct to $4 \mathrm{~km} \mathrm{~s}^{-1}$.

We observed the Balmer lines $\mathrm{H} \gamma(4340.46 \AA), \mathrm{H} \delta$ $(4101.73 \AA), \mathrm{H} \zeta(3889.05 \AA)$ and $\mathrm{H} \eta(3835.38 \AA)$ near maximum visible light when possible in order to get the shock

\footnotetext{
1 http://www.kusastro.kyoto-u.ac.jp/vsnet/gcvs/
} 
velocity deep in the atmosphere. Between maximum and minimum light we searched for emission lines of the metals MgI, Si I, MnI, FeI and FeII. Table 2 lists the multiplet number $M$, wavelength $\lambda$, upper and lower energy levels of the transition $E_{\mathrm{u}}$ and $E_{\mathrm{l}}$, and the Einstein-coefficients $A_{\mathrm{ul}}$ for the metal emission lines observed. This data was obtained from the NIST database ${ }^{2}$ (Fuhr et al. 1988). Note that the detected lines include the forbidden lines of the multiplets $[\mathrm{Fe} \mathrm{II}] 6 \mathrm{~F}$, [Fe II] $7 \mathrm{~F}$ and $[\mathrm{Fe} \mathrm{II}] 21 \mathrm{~F}$.

Table 2. Metal emission line properties

\begin{tabular}{llllll}
\hline Ion & $\mathrm{M}$ & $\lambda[\AA]$ & $E_{\mathrm{u}}\left[\mathrm{cm}^{-1}\right]$ & $E_{\mathrm{l}}\left[\mathrm{cm}^{-1}\right]$ & $A_{\mathrm{ul}}\left[\mathrm{s}^{-1}\right]$ \\
\hline Si I & 2 & 4102.95 & 39760 & 15394 & $9.6+4$ \\
Mn I & 2 & 4030.75 & 24802 & 0 & $1.7+7$ \\
Mg I & 1 & 4571.10 & 21870 & 0 & $2.2+2$ \\
& 3 & 3829.32 & 47957 & 21850 & $8.9+7$ \\
& 3 & 3832.35 & 47957 & 21870 & $6.7+7$ \\
& 3 & 3838.29 & 47957 & 21911 & $4.5+6$ \\
Fe I & 2 & 4461.65 & 23111 & 704 & $2.9+4$ \\
& 2 & 4375.93 & 22846 & 0 & $2.9+4$ \\
& 3 & $4206.70^{*}$ & 24181 & 416 & $7.2+3$ \\
& 3 & 4216.18 & 23711 & 0 & $1.8+4$ \\
& 3 & $4291.46^{*}$ & 23711 & 416 & $4.1+3$ \\
& 42 & 4307.90 & 35768 & 12560 & $3.4+7$ \\
& 42 & 4202.03 & 35768 & 11976 & $8.2+6$ \\
& 73 & 3852.57 & 32499 & 17550 & $2.9+6$ \\
& 648 & $4374.49^{*}$ & 49477 & 26624 & $4.9+5$ \\
Fe II & 38 & 4427.30 & 22997 & 416 & $3.4+4$ \\
& $6 \mathrm{~F}$ & 4583.84 & 44449 & 22637 & $3.8+5$ \\
& $7 \mathrm{~F}$ & 4359.33 & 22810 & 385 & $2.9-1$ \\
& $7 \mathrm{~F}$ & 4287.39 & 23318 & 385 & $1.1+0$ \\
& $21 \mathrm{~F}$ & 4276.83 & 25805 & 0 & $1.5+0$ \\
& $21 \mathrm{~F}$ & 4243.97 & 25429 & 1872 & $6.5-1$ \\
\hline
\end{tabular}

* Searched for, but not observed in our spectra.

For data reduction, the Image Reduction and Analysis Facility (IRAF) of the National Optical Astronomy Observatories was used. The wavelength calibration of the spectra was obtained from thorium-argon arcs taken during each run. The standard stars HR 718, HR 3454 or HR 7596 were observed on photometric nights to allow calibration of the spectra to absolute fluxes. The calibrated fluxes from multiple observations of standard stars during each night were compared to estimate the errors in the fluxes tabulated in Tables 4-8: these comparisons indicate that the flux errors should be less than $5 \%$. This error is much less than the typical variation of flux throughout the pulsation cycle (see Sect. 4).

In the case of non-photometic nights, we had to estimate the absolute fluxes from the spectra by assuming that the flux in the $V$ band at $5100 \AA$ (a quasi-continuum point) varies in the same way as the light curves of The American Association Of Variable Star Observers

\footnotetext{
${ }^{2}$ http://physics.nist.gov/cgi-bin/AtData/main_asd
}

$(\text { AAVSO) })^{3}$ (Mattei et al. 1980). With this method we were able to estimate the continuum flux at $5100 \AA$ by scaling the non-photometric flux relative to the calibrated flux from a photometric night. We were then able to calibrate the flux at other wavelengths using the relative spectral response of the spectrograph. The line fluxes obtained in this way are marked with a colon in Tables 4-8.

In order to derive the emission line fluxes, it is necessary to have an estimate of the continuum level. This was estimated by eye: initially, the whole order containing the line was examined to get an idea of the overall continuum shape, then a wavelength interval on either side of the line approximately equal to the line width was used for the final estimate. In the case of P-Cygni line profiles, the associated absorption was ignored in the continuum estimate. For strong emission lines (ex. Balmer lines), the continuum level is not a significant contributor to line flux errors, but in the case of weak emission lines in these stars which exhibit many absorption lines (ex. Fe I $4307.90 \AA$ at phase 0.63 in R Leo, Fig. 16), the continuum level can be a major contributor to the flux error. In the worst cases, we estimate that the flux could be wrong by a factor of 2 .

The phases for each observation of each Mira variable were determined from the AAVSO maxima: phase zero corresponds to visible maximum. Table 3 lists the Julian dates and the corresponding phases for the variables observed.

Table 3. Julian dates and phases of observation

\begin{tabular}{lrrrrrr}
\hline JD $^{a}$ & & \multicolumn{5}{c}{ Phase } \\
& RR Sco & R Aql & R Car & R Leo & S Scl & R Hya \\
\hline 1153 & - & - & -0.16 & - & - & - \\
1169 & - & - & - & - & 0.02 & - \\
1206 & - & - & -0.09 & 0.43 & - & - \\
1268 & - & 0.01 & 0.11 & 0.63 & - & - \\
1357 & 0.29 & 0.32 & 0.39 & - & 0.52 & 0.08 \\
1375 & - & 0.39 & 0.45 & - & 0.58 & 0.12 \\
1412 & 0.48 & 0.50 & 0.57 & - & 0.67 & 0.21 \\
1440 & 0.58 & - & 0.66 & - & 0.75 & - \\
1464 & - & 0.69 & - & - & - & - \\
1542 & - & - & - & 1.49 & - & 0.53 \\
\hline
\end{tabular}

a Julian date -2450000 .

\section{The emission line spectra}

\subsection{Hydrogen lines}

Observed line profiles for the Balmer lines $\mathrm{H} \gamma, \mathrm{H} \delta, \mathrm{H} \zeta$ and $\mathrm{H} \eta$ are presented in Figs. 1-5 for RR Sco, R Aql, R Car, S Scl and R Hya (because of its position in the sky and the phase of its light curve, R Leo was not observable near maximum when the Balmer lines were in emission, but we did observe it during two different minima). The line

\footnotetext{
${ }^{3}$ http://www. aavso.org/adata/curvegenerator.shtml
} 

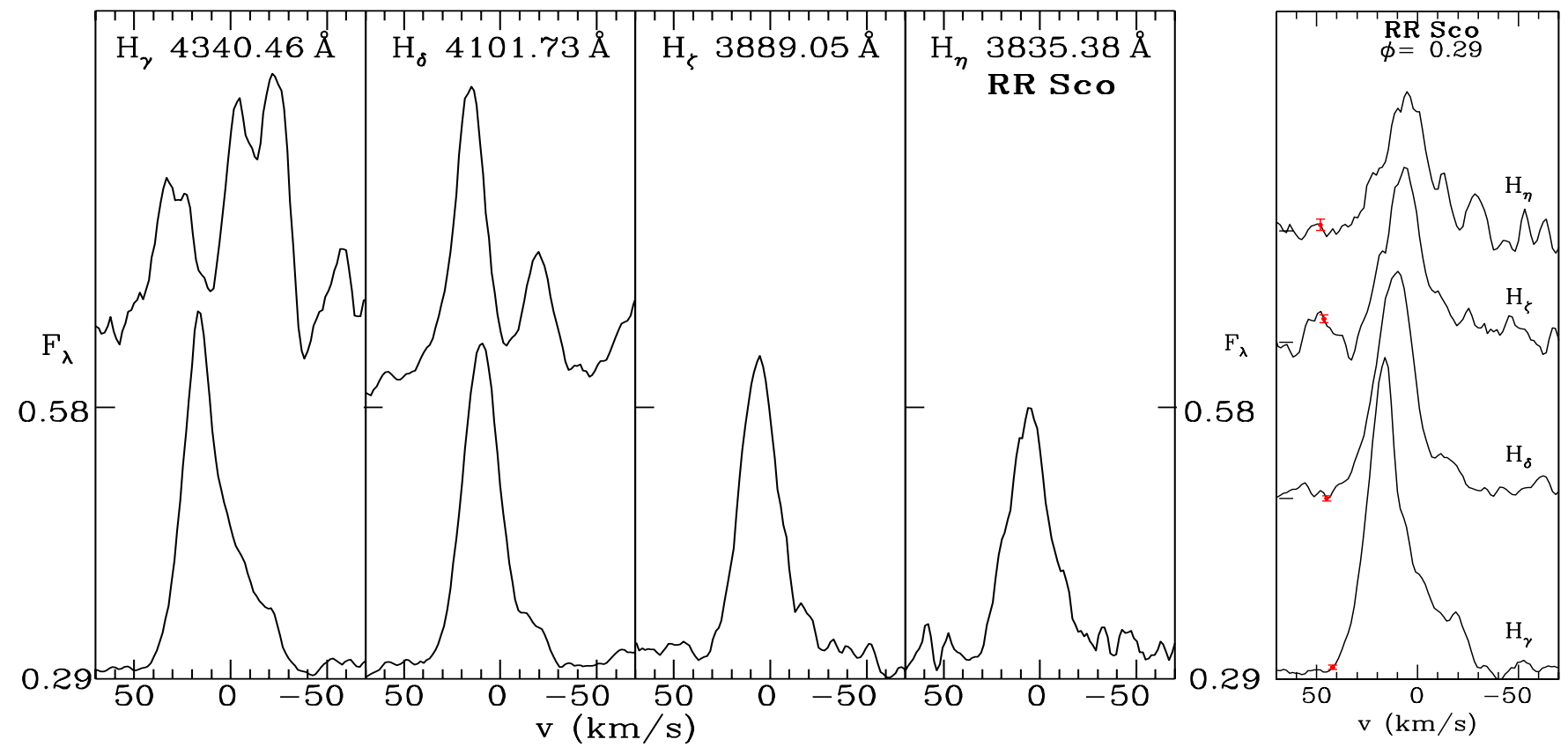

Fig. 1. Left: Line profiles of $\mathrm{H} \gamma, \mathrm{H} \delta, \mathrm{H} \zeta, \mathrm{H} \eta$ as a function of phase in RR Sco. The observations were made with a wide (1500 $\mu$ m) slit. $\mathrm{F}_{\lambda}$ is plotted vertically, with the zero flux level for each spectrum being indicated by a tick mark on the vertical axis. The phase of the observation is indicated next to each tick mark. Note that the height of the profiles is arbitrary as we scaled them with different factors. Table 4 lists the maximum flux level in each line. The horizontal axis corresponds to wavelength $\lambda$ (i.e. velocity). The spectra have been positioned horizontally so that line emission in the rest frame of the star will appear at zero velocity (stellar center-of-mass velocities from Table 1 have been adopted). Positive velocities correspond to motion outward from the center-of-mass (i.e. $\lambda$ increases to the right). Right: The Balmer line profiles observed with a narrow (300 $\mu \mathrm{m})$ slit at phase $\phi=0.29$, shown for comparison with the wide slit observations
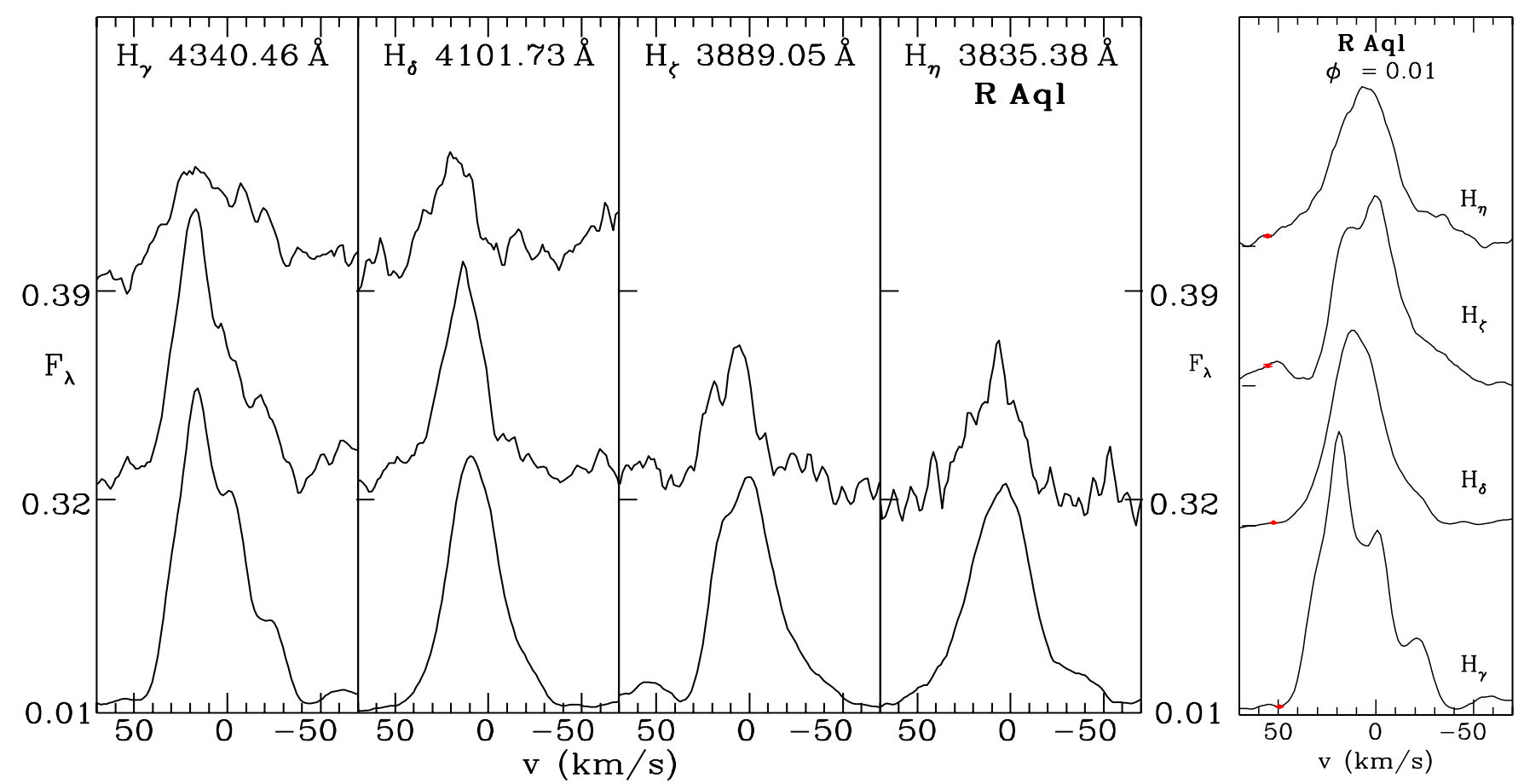

Fig. 2. Left: Line profiles of $\mathrm{H} \gamma, \mathrm{H} \delta, \mathrm{H} \zeta, \mathrm{H} \eta$ as a function of phase in R Aql (see Fig. 1 for details). Right: The Balmer line profiles observed with a narrow $(300 \mu \mathrm{m})$ slit at phase $\phi=0.01$ 


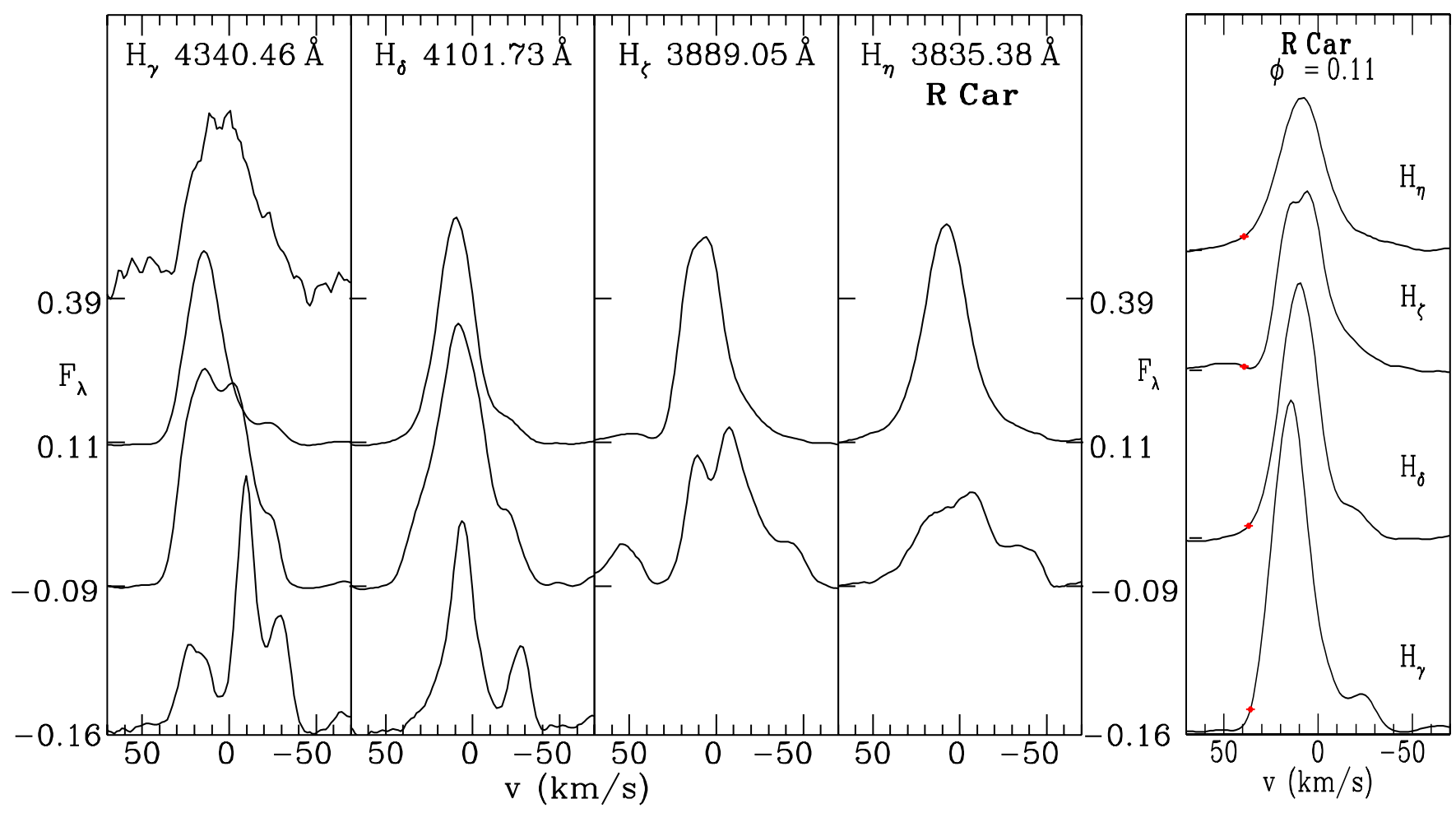

Fig. 3. Left: Line profiles of $\mathrm{H} \gamma, \mathrm{H} \delta, \mathrm{H} \zeta, \mathrm{H} \eta$ as a function of phase in R Car (see Fig. 1 for details). Right: The Balmer line profiles observed with a narrow $(300 \mu \mathrm{m})$ slit at phase $\phi=0.11$

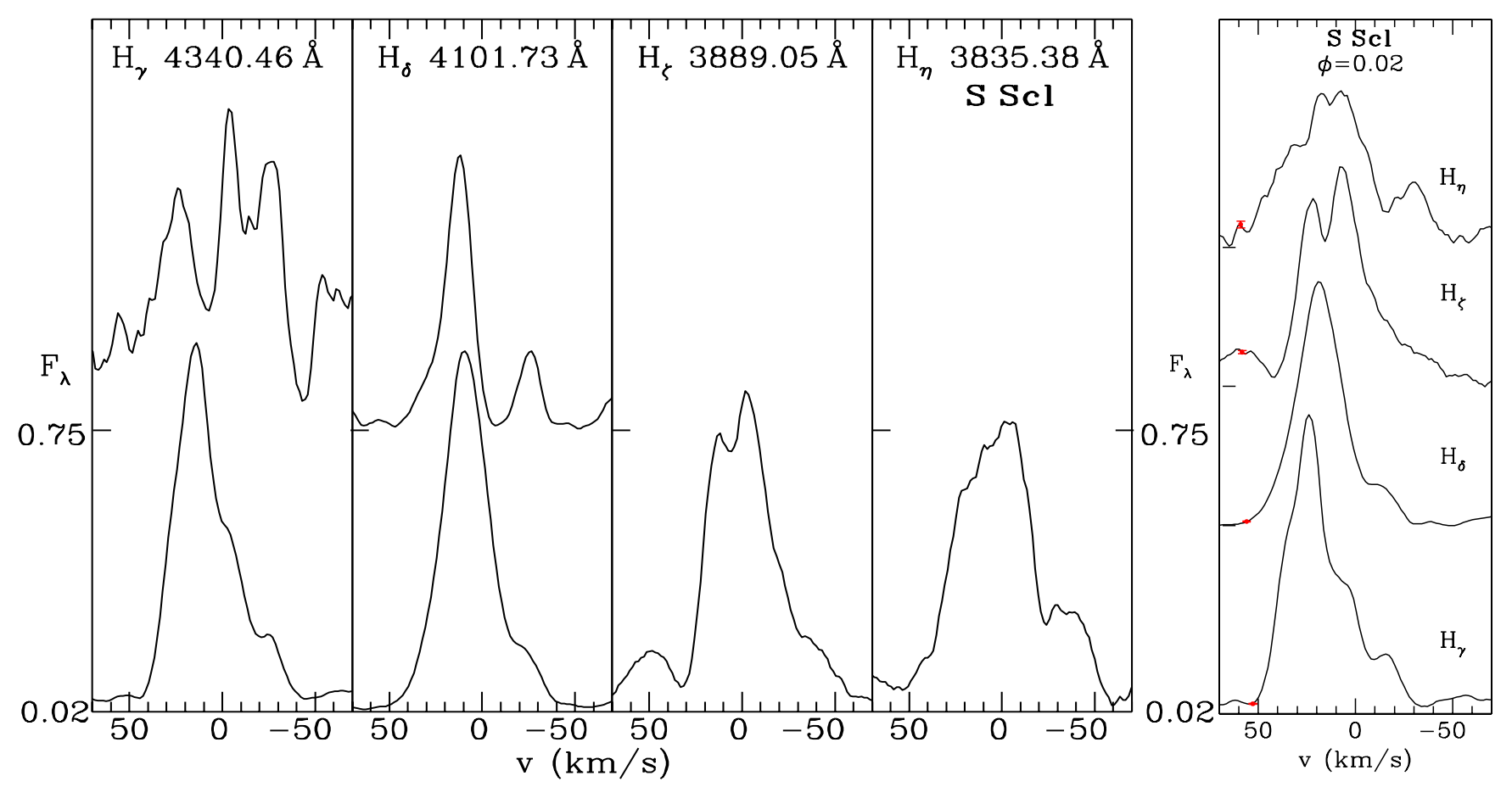

Fig. 4. Left: Line profiles of $\mathrm{H} \gamma, \mathrm{H} \delta, \mathrm{H} \zeta, \mathrm{H} \eta$ as a function of phase in S Scl (see Fig. 1 for details). Right: The Balmer line profiles observed with a narrow $(300 \mu \mathrm{m})$ slit at phase $\phi=0.02$ 

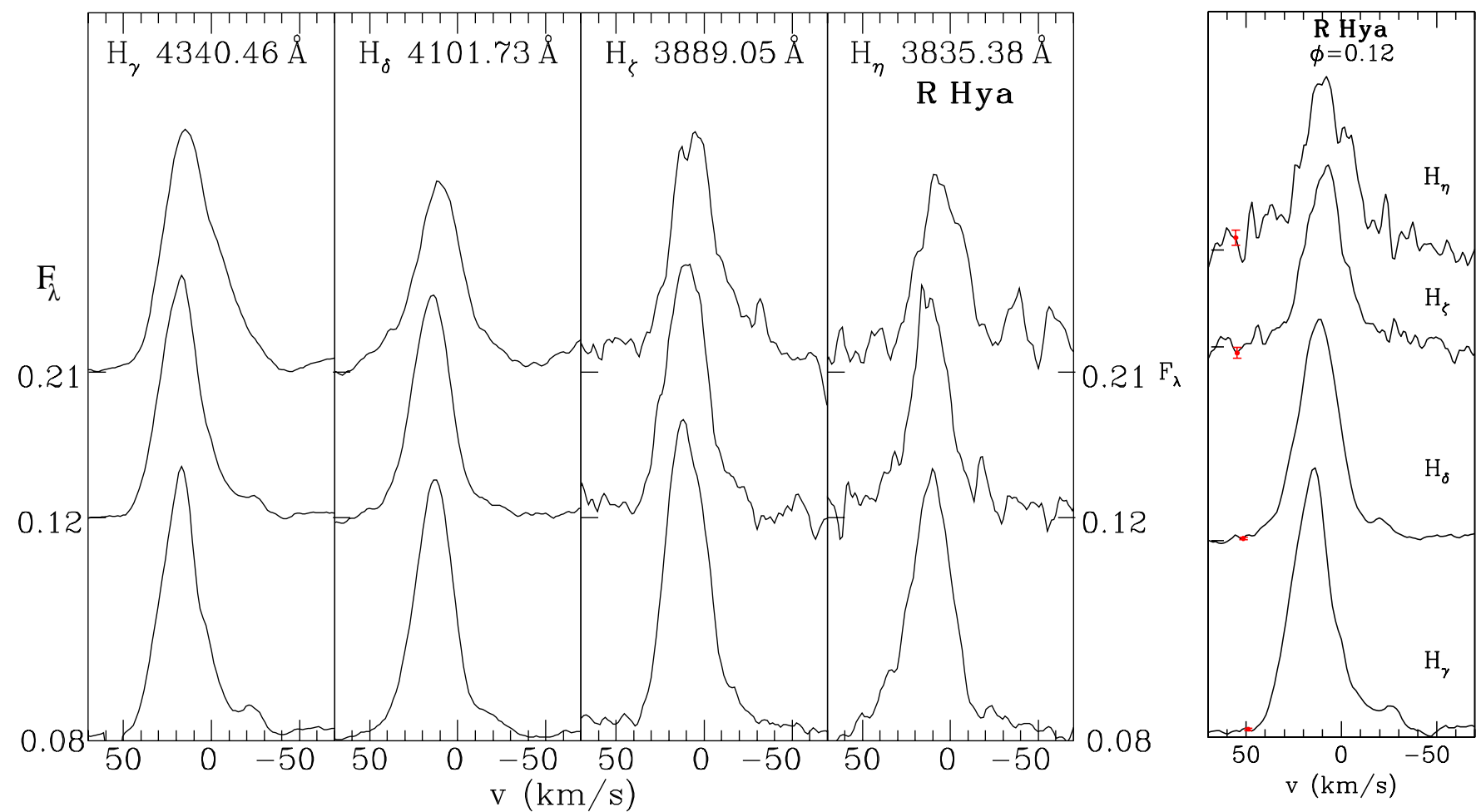

Fig. 5. Left: Line profiles of $\mathrm{H} \gamma, \mathrm{H} \delta, \mathrm{H} \zeta, \mathrm{H} \eta$ as a function of phase in R Hya (see Fig. 1 for details). Right: The Balmer line profiles observed with a narrow $(300 \mu \mathrm{m})$ slit at phase $\phi=0.12$
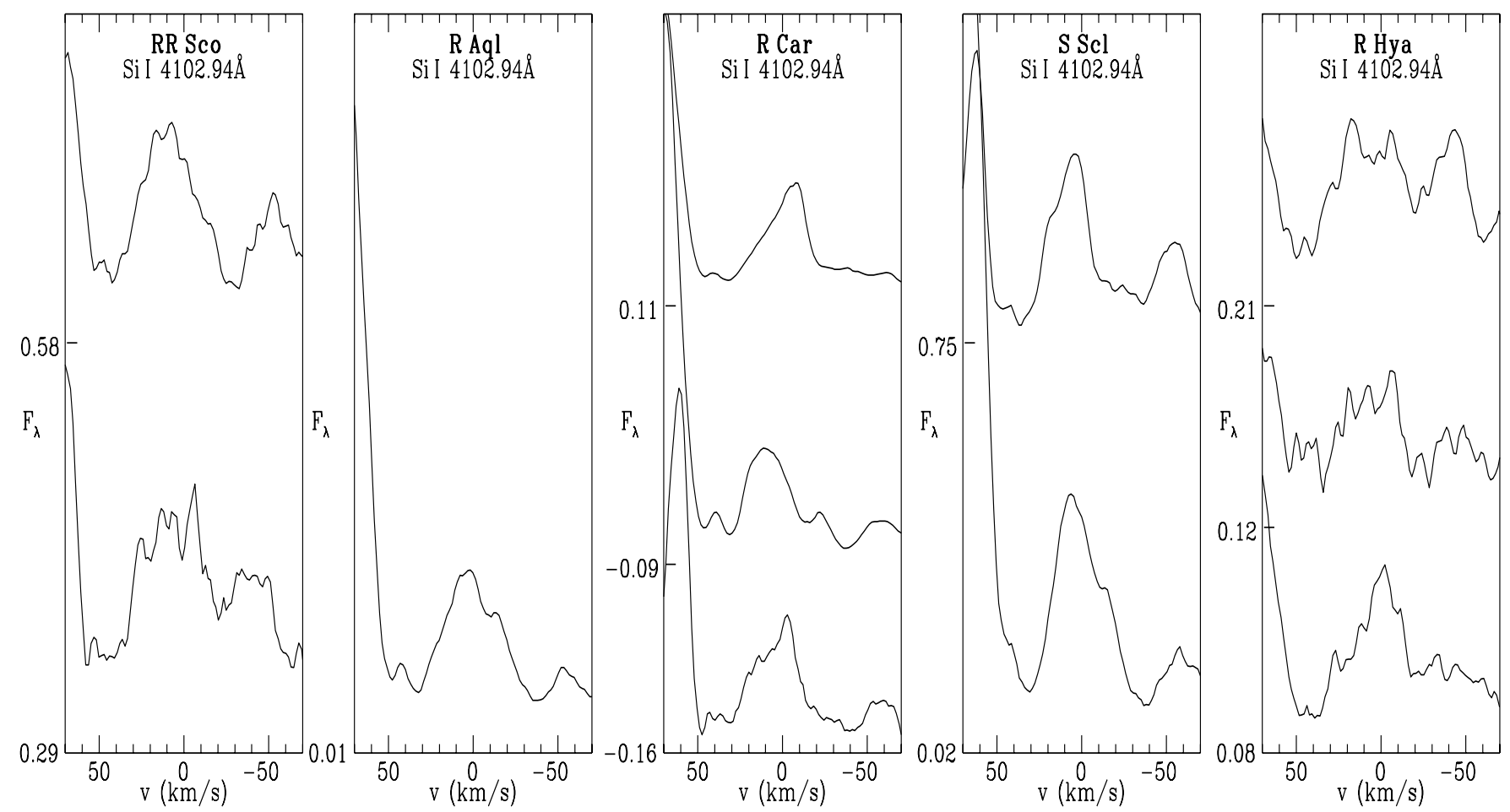

Fig. 6. From left to right: Line profiles of Si I $4102.94 \AA$ (M 2) as a function of phase in RR Sco, R Aql, R Car, S Scl and R Hya (plotted as in the left panel of Fig. 1). The feature on the left of each plot is the edge of the nearby H $\delta$ emission line. Table 4 lists the maximum flux level in each Si I line 

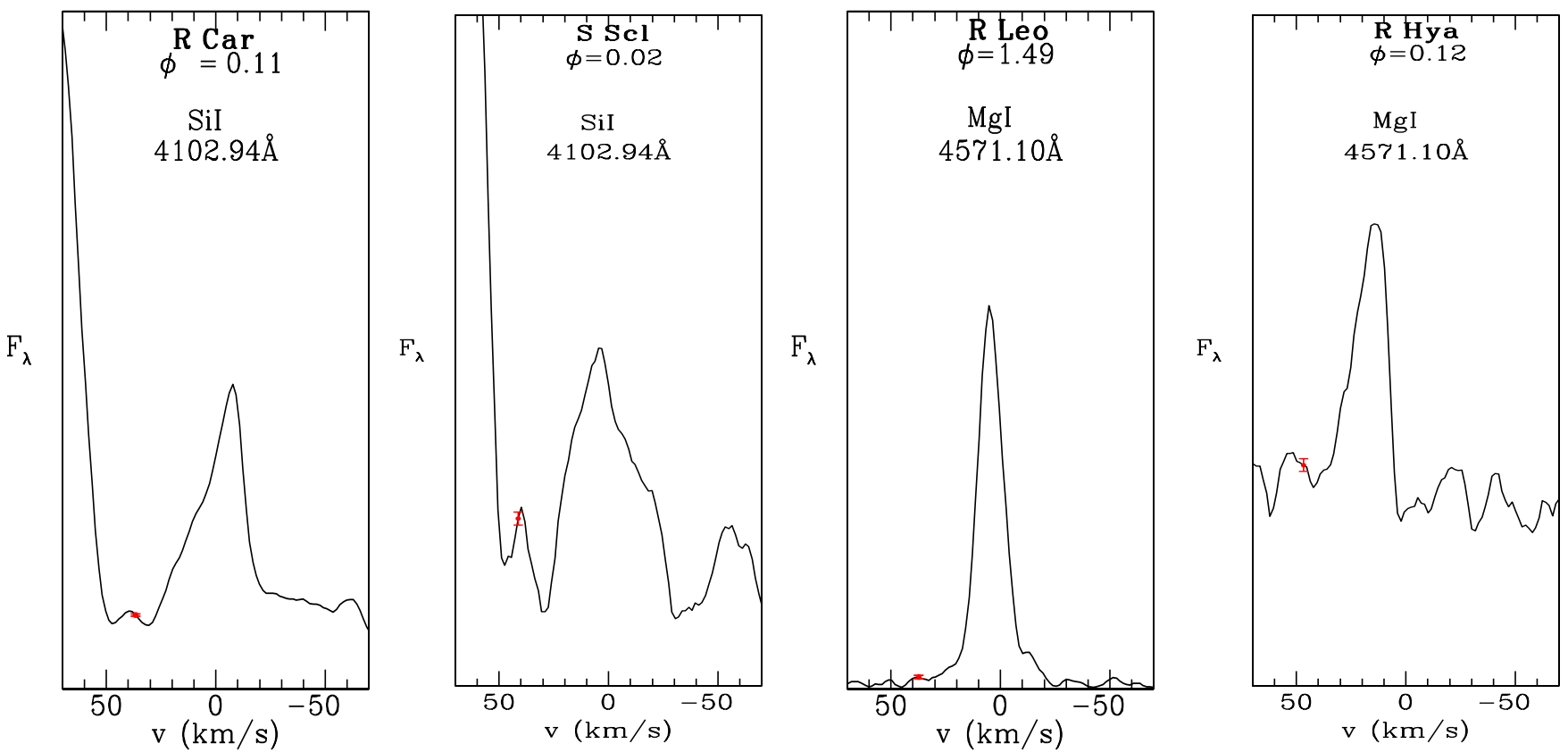

Fig. 7. From left to right: Narrow $(300 \mu \mathrm{m})$ slit spectra for the Si I $4102.94 \AA$ line at phase $\phi=0.11$ in $\mathrm{R}$ Car and $\phi=0.02$ in $\mathrm{S} \mathrm{Scl}$, and the Mg I $4571.10 \AA$ line at phase $\phi=1.49$ in R Leo and $\phi=0.12$ in R Hya. These plots are shown for comparison with the wide $(1500 \mu \mathrm{m})$ slit observations in Figs. 6 and 8
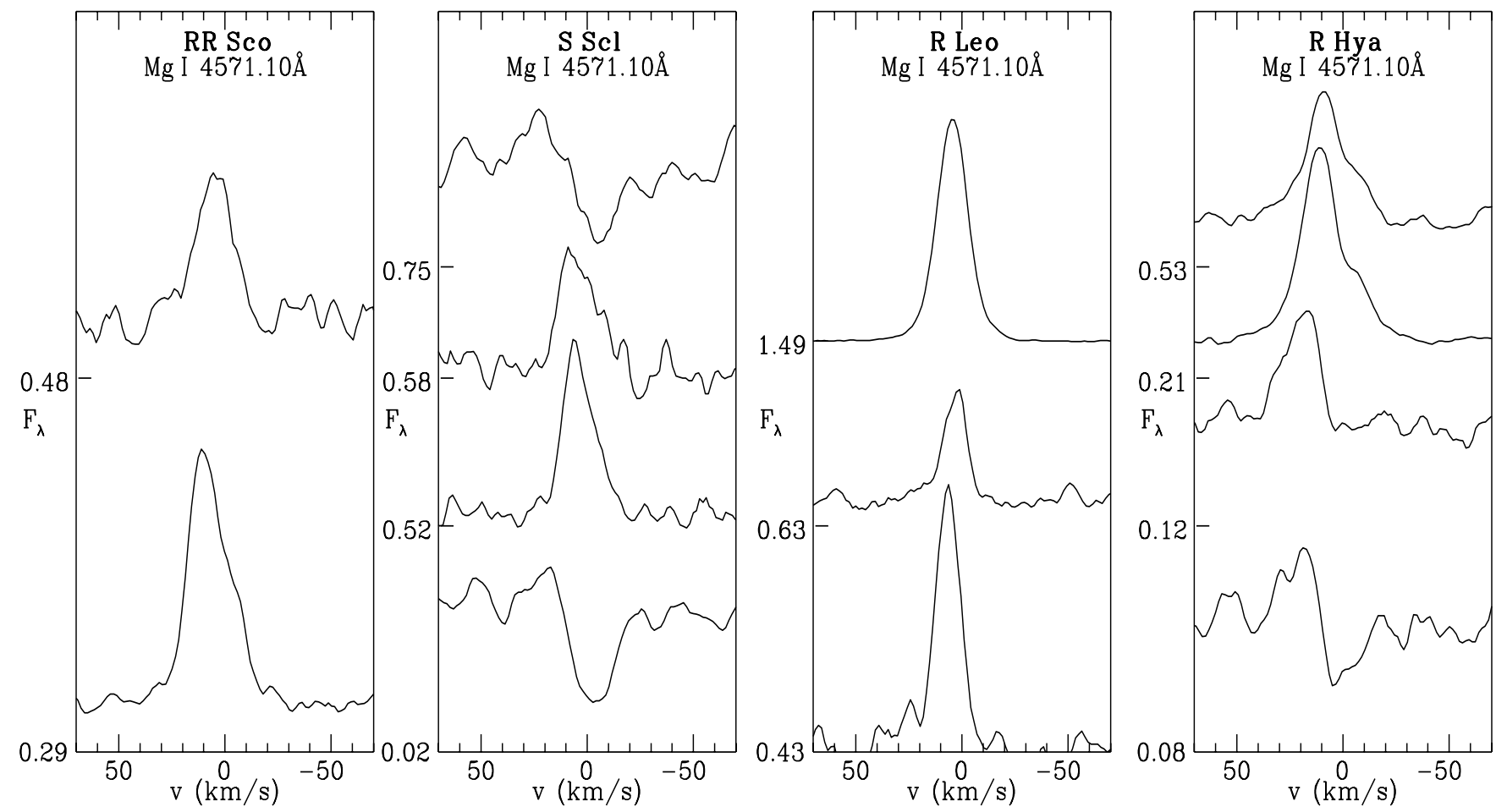

Fig. 8. From left to right: Line profiles of Mg I $4571.10 \AA$ (M 1) as a function of phase in RR Sco, S Scl, R Leo and R Hya (plotted as in the left panel of Fig. 1). Table 5 lists the maximum flux level in each line 

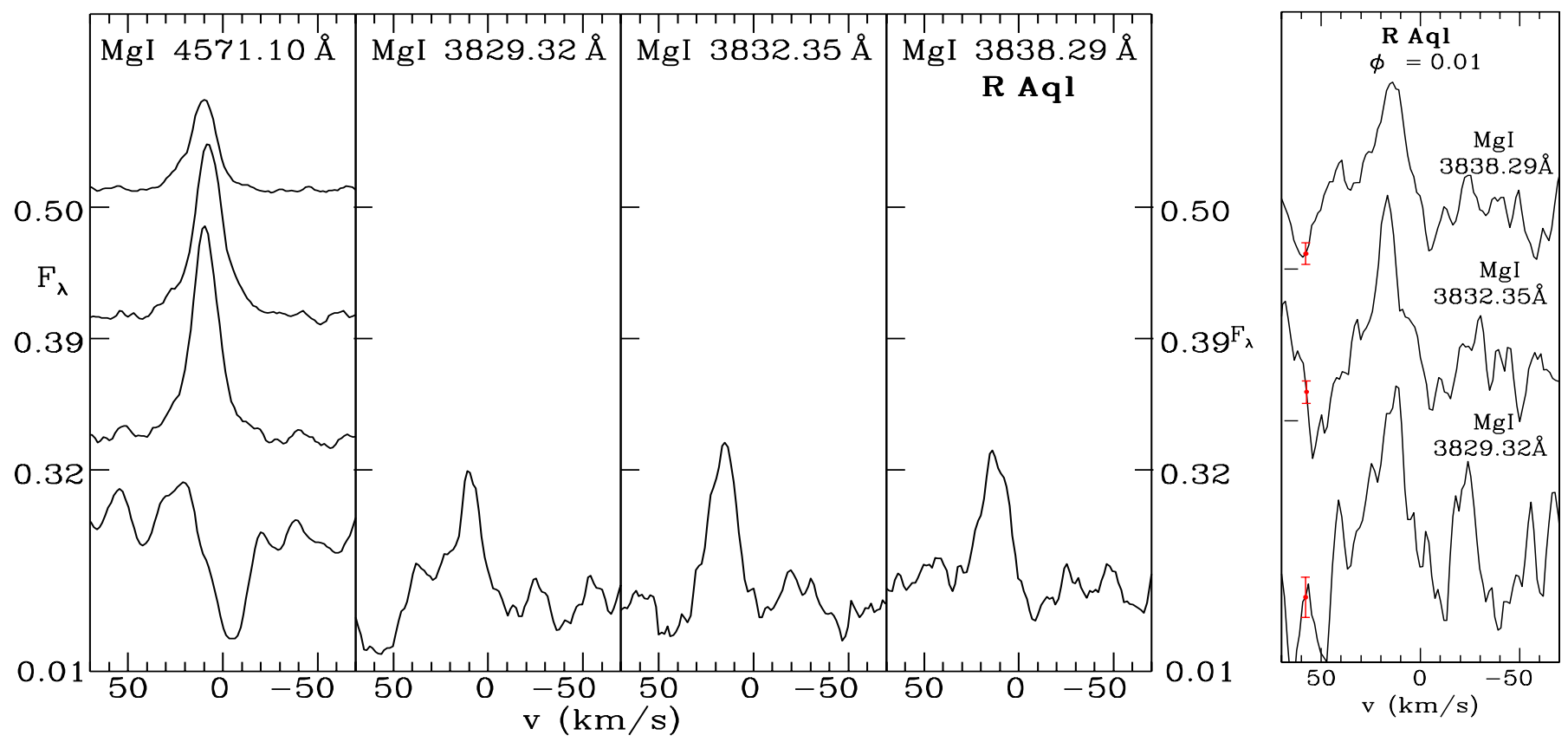

Fig. 9. Left: Line profiles of Mg I 4571.10 $\AA$ (M 1) and Mg I 3829.32 $\AA, 3832.35 \AA$ and $3838.29 \AA$ (all M 3) as a function of phase in R Aql (plotted as in the left panel of Fig. 1). Table 5 lists the maximum flux level in each line. Right: Profiles of the Mg I multiplet 3 lines observed with a narrow slit $(300 \mu \mathrm{m})$ at a single phase, shown for comparison with the wide slit (1500 $\mu \mathrm{m})$ observations. $F_{\lambda}$ is plotted vertically, with the zero flux level for each spectrum being indicated by a tick mark on the vertical axis
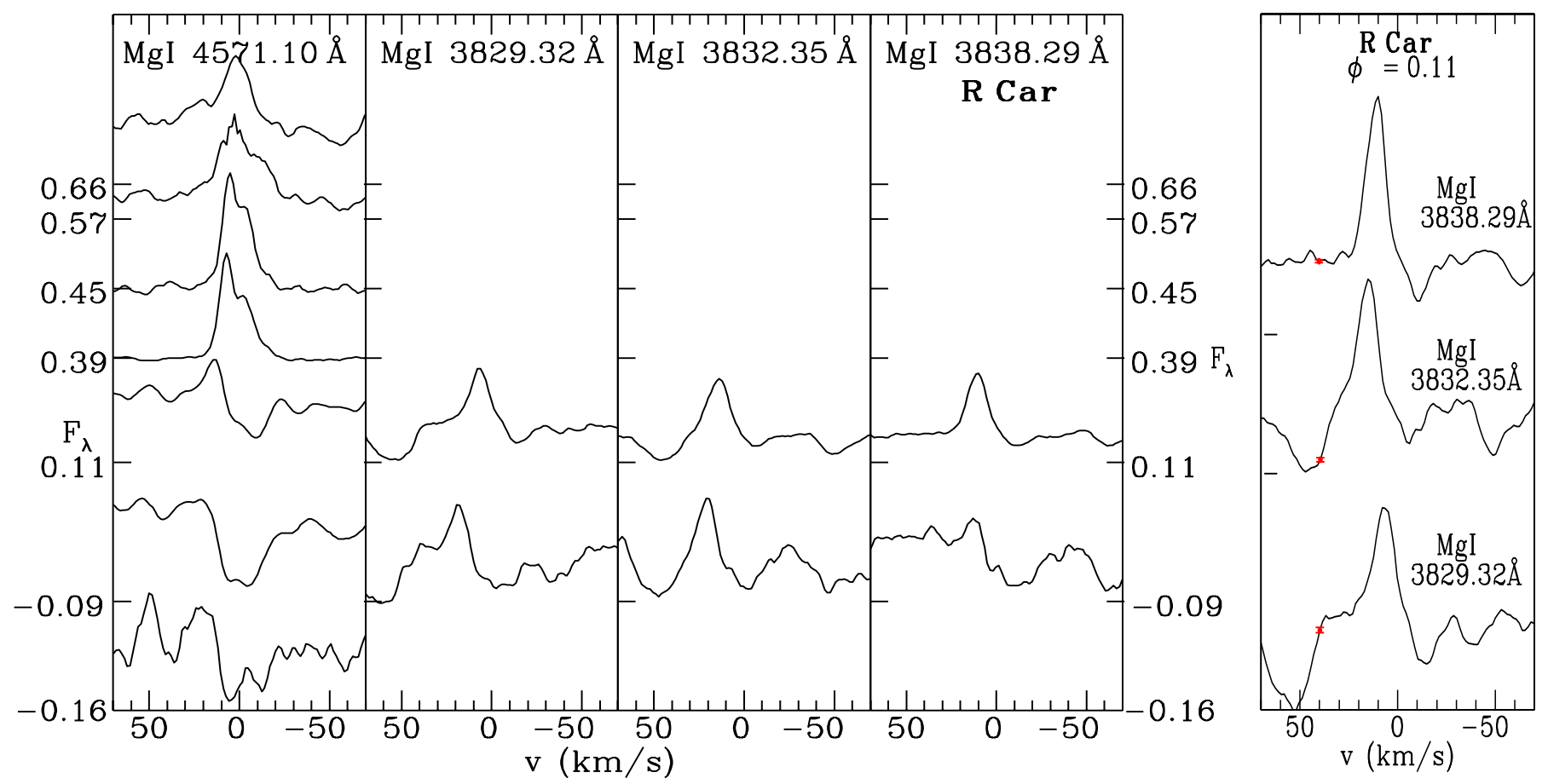

Fig. 10. Same as Fig. 9 but for R Car 


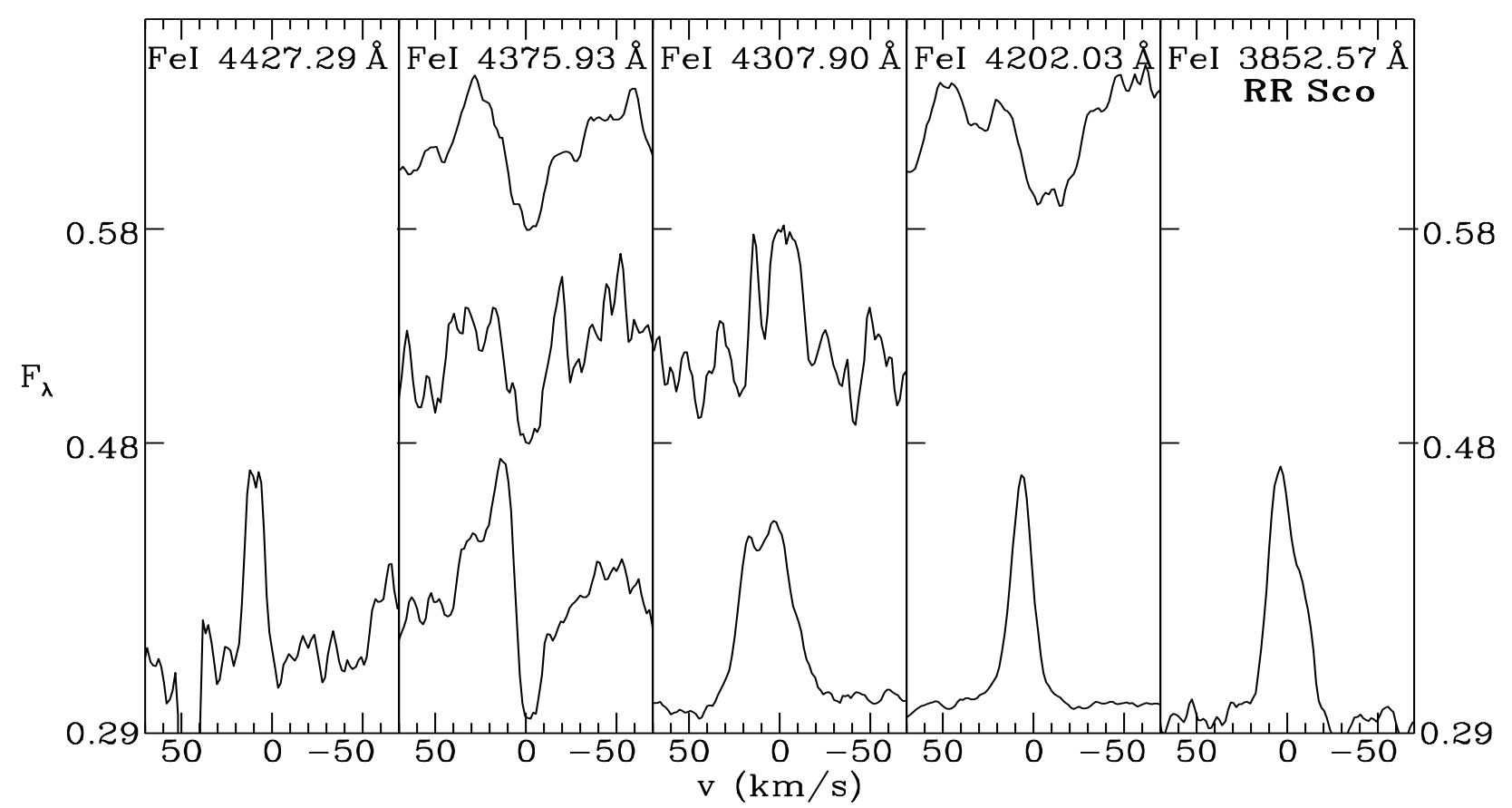

Fig. 11. Line profiles of FeI 4427.30 A (M 828), 4375.93 $\mathrm{A}$ (M 2), $4307.90 \AA, 4202.03 \AA$ (both M 42$)$ and $3852.57 \AA$ (M 73 ) as a function of phase in RR Sco (plotted as in the left panel of Fig. 1). Tables 6 and 7 list the maximum flux level in each line

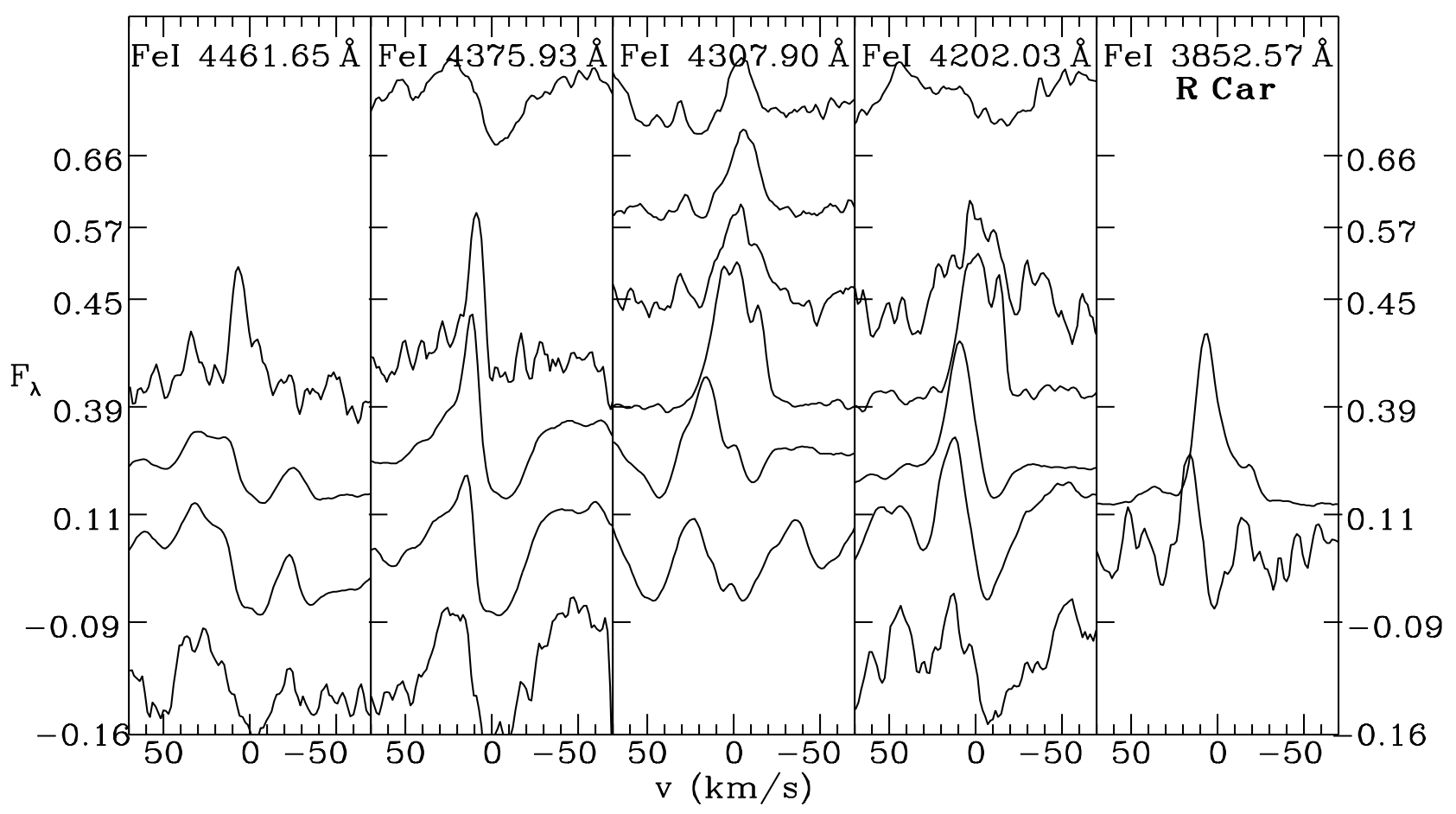

Fig. 12. Same as Fig. 11 but for R Car, and with Fe I 4461.65 $\AA$ (M 2) replacing Fe I $4427.30 \AA$ (M 828) in the left panel 

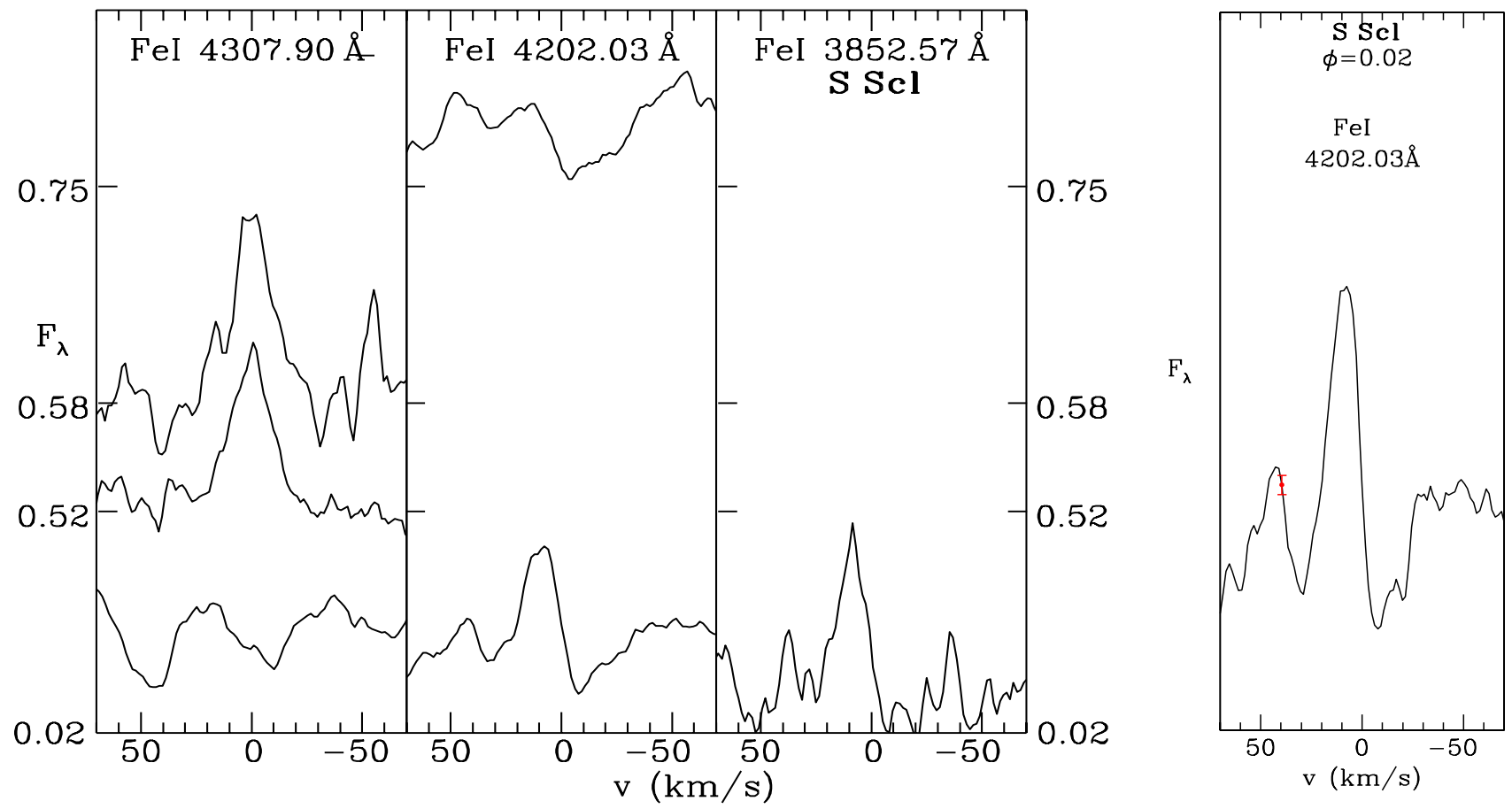

Fig. 13. Left: Line profiles of Fe I $4307.90 \AA, 4202.03 \AA$ (both M 42) and $3852.57 \AA$ (M 73 ) as a function of phase in S Scl (plotted as in the left panel of Fig. 1). Tables 6 and 7 list the maximum flux level in each line. Right: The FeI $4202.03 \AA$ line profile observed with a narrow $(300 \mu \mathrm{m})$ slit at phase $\phi=0.02$, shown for comparison with the wide slit $(1500 \mu \mathrm{m})$ observations
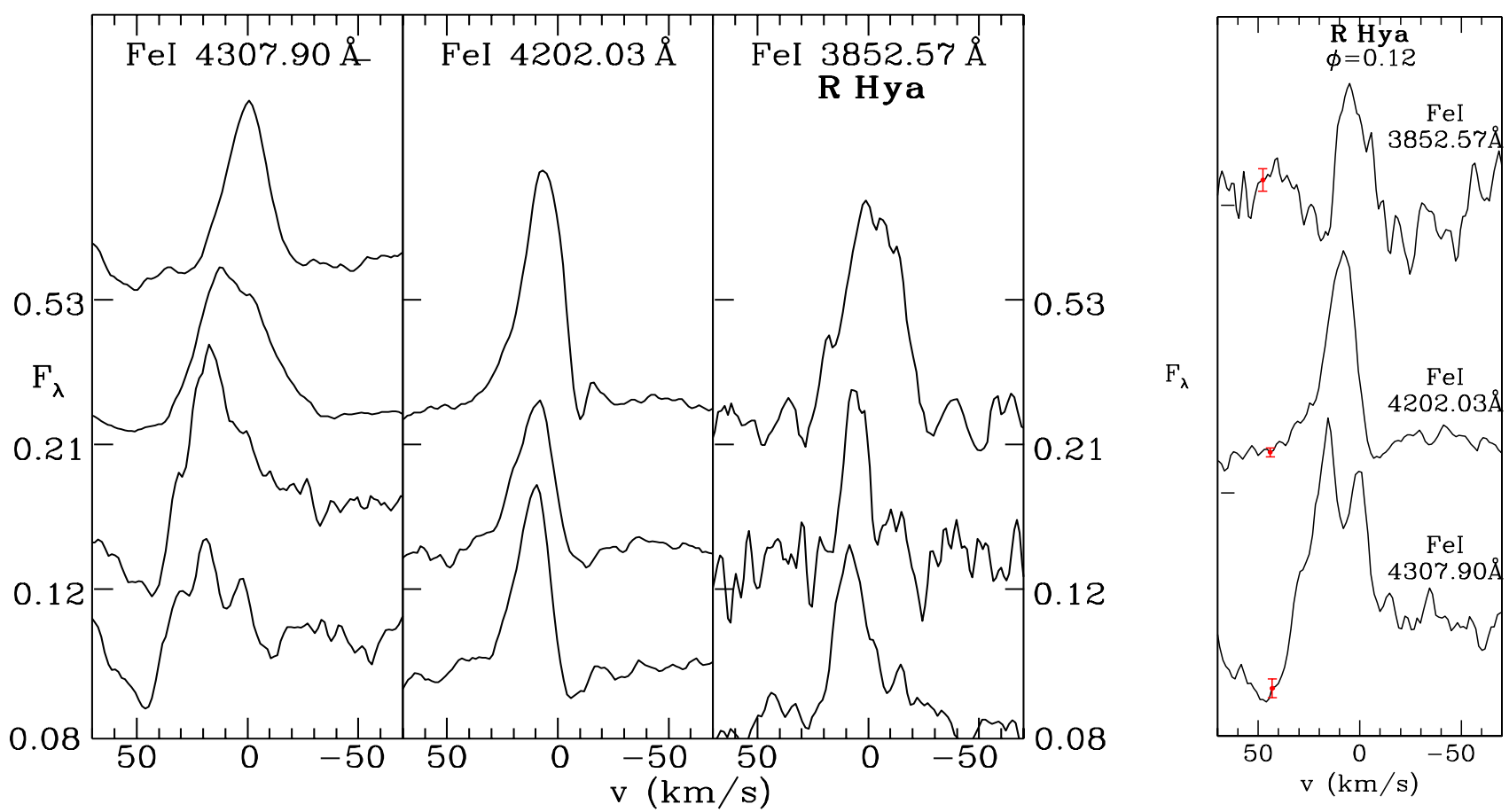

Fig. 14. Left: Same as Fig. 13 but for R Hya, and including Fe I $4307.90 \AA$ (M 42) and $3852.57 \AA$ (M 73) in the right panel 

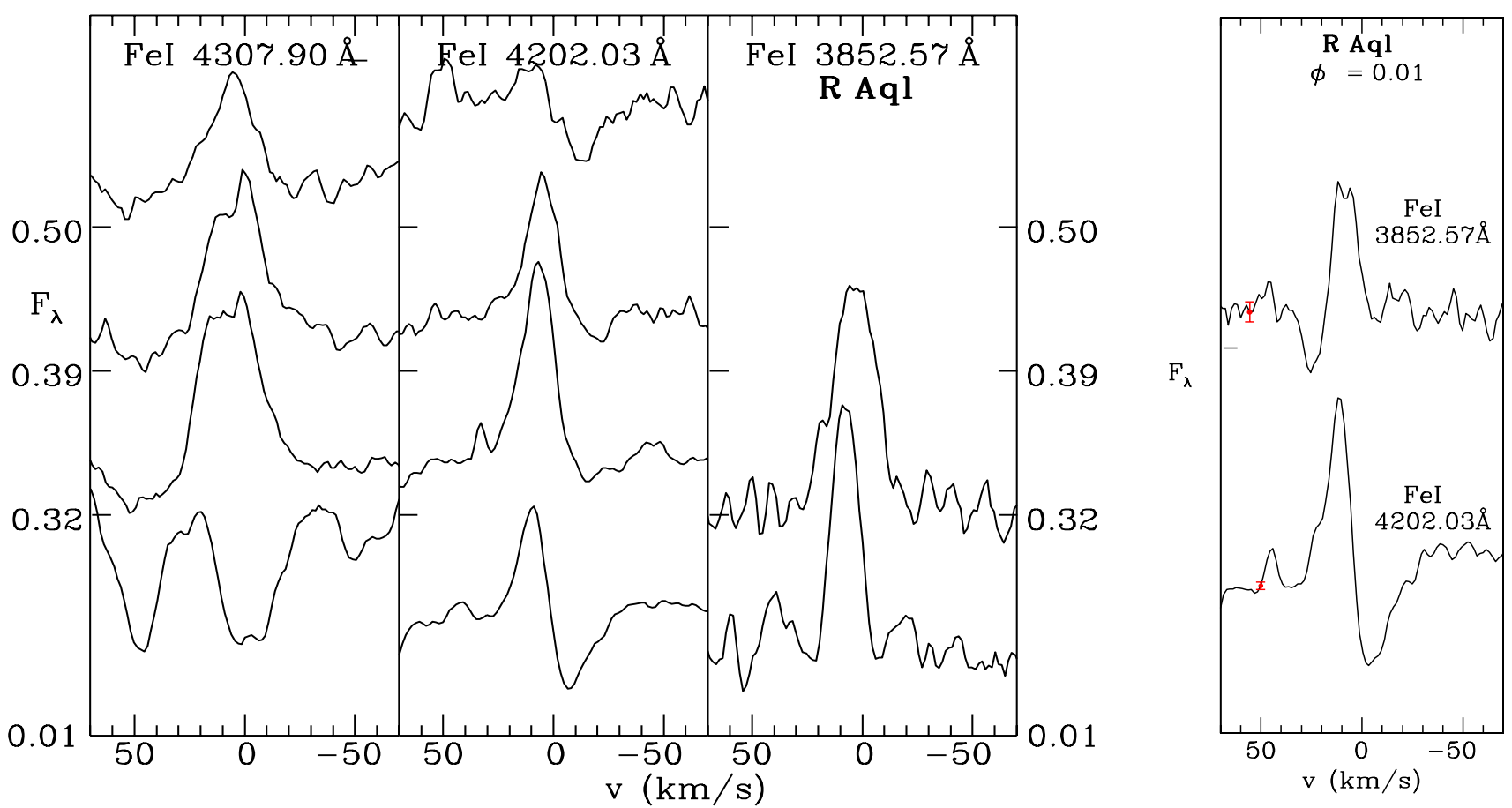

Fig. 15. Left: Same as Fig. 13 but for R Aql, and including FeI $3852.57 \AA$ (M 73) in the right panel
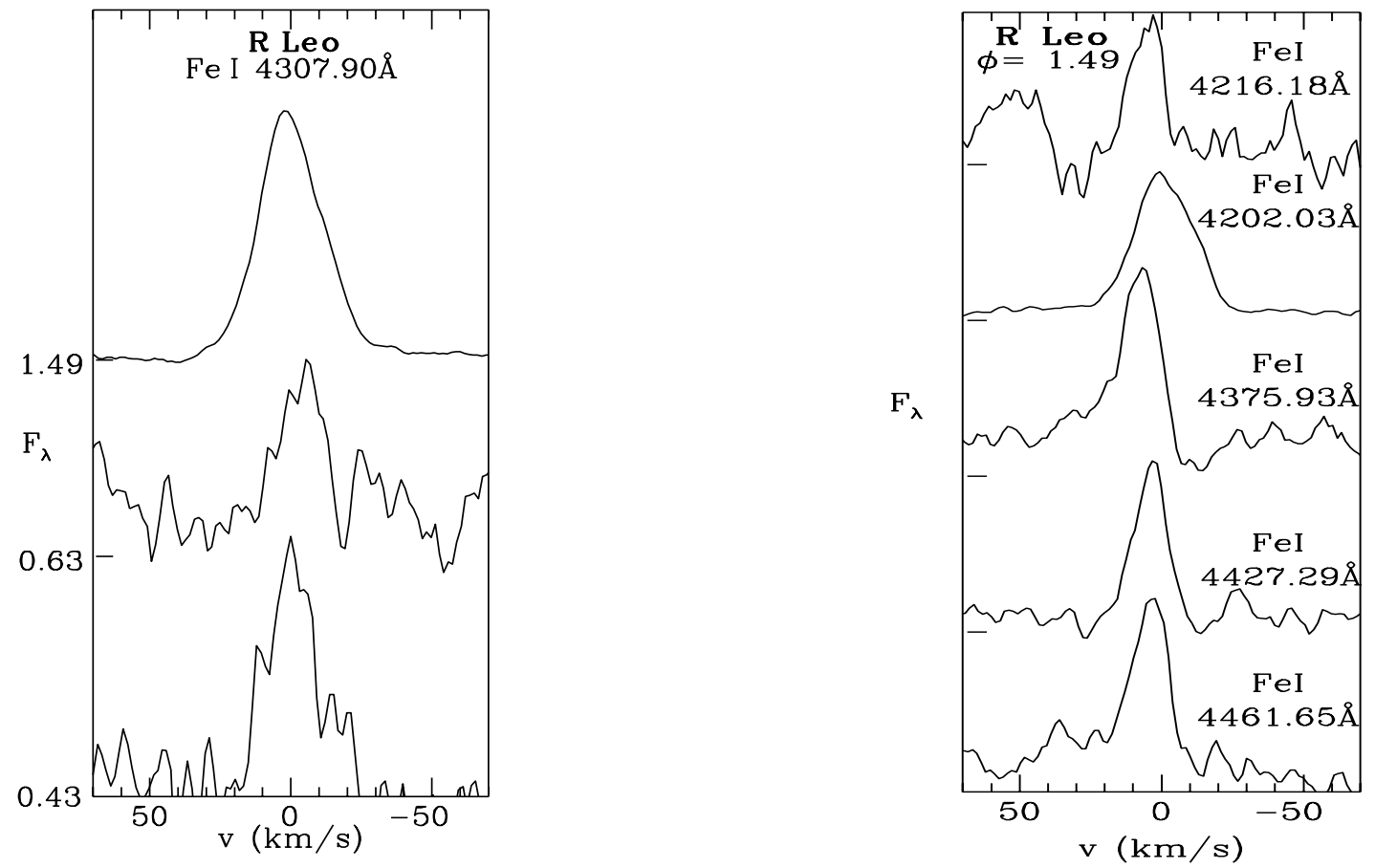

Fig. 16. Left: Line profiles of Fe I $4307.90 \AA$ (M 42) as a function of phase in R Leo. Right: Line profiless of Fe I $4461.65 \AA$, $4375.93 \AA$ (both M 2), 4427.30 $\AA$ (M 828), 4202.03 $\mathrm{A}$ (M 42) and 4216.18 $\mathrm{A}$ (M 3) at phase $\phi=1.49$ in R Leo. Both panels are plotted as in the left panel of Fig. 1. Tables 6 and 7 list the maximum flux level in each line 

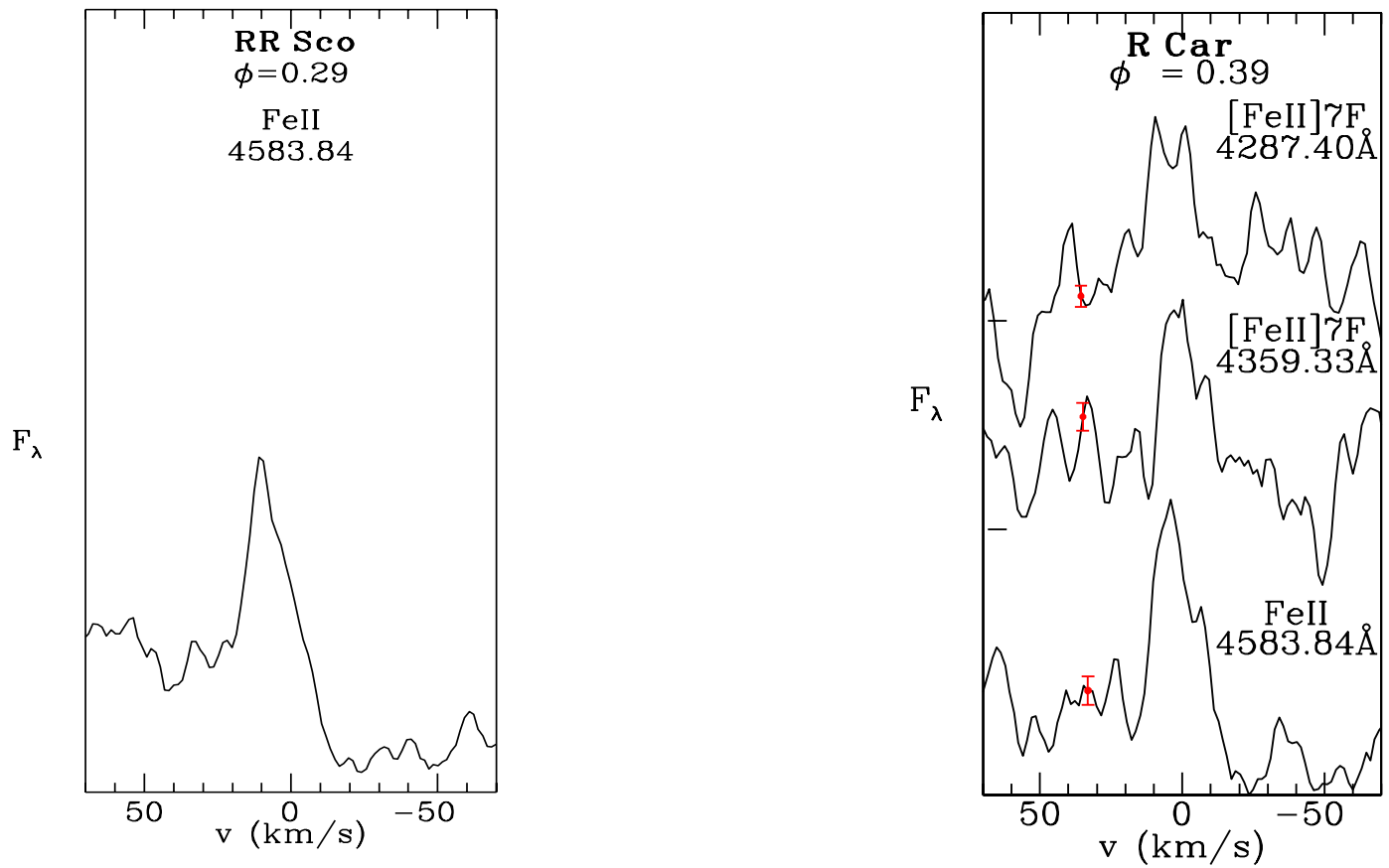

Fig. 17. Left: Line profile of Fe II $4583.84 \AA$ (M 38) at phase $\phi=0.29$ in RR Sco $(1500 \mu \mathrm{m})$. Table 7 lists the maximum flux level in the line. Right: Line profiles of Fe II 4583.84 $($ M 38) and of the forbidden transitions [Fe II] 7F (4359.33 $\mathrm{A}$ and $4287.40 \AA$ ) at phase $\phi=0.39$ in R Car. $F_{\lambda}$ is plotted vertically, with the zero flux level for each spectrum being indicated by a tick mark on the vertical axis. Table 8 lists the maximum flux level in each line
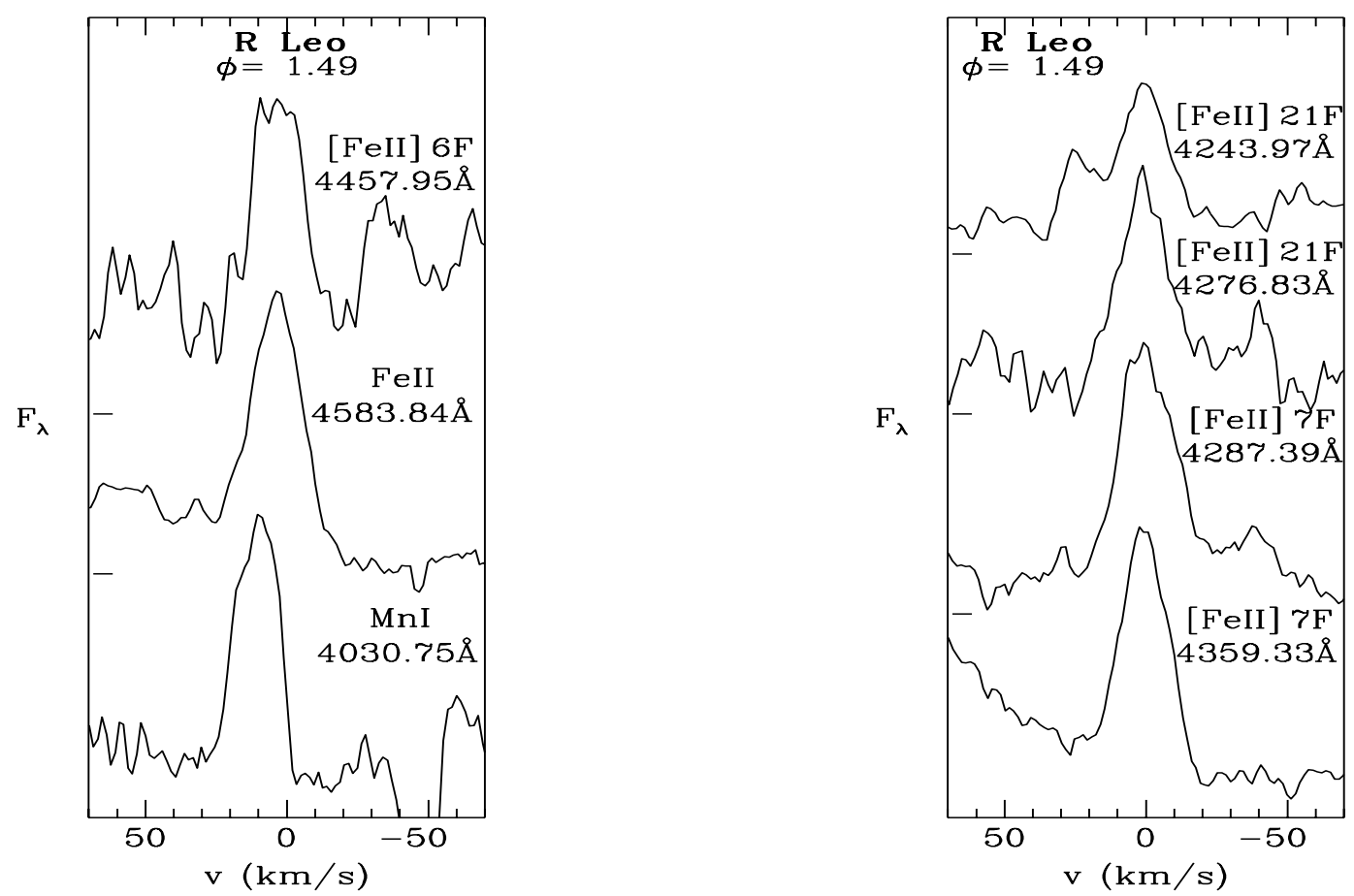

Fig. 18. Left: Line profiles of Mn I $4030.75 \AA$ (M 2), Fe II $4583.84 \AA$ (M 38) and [Fe II] $6 \mathrm{~F} 4457.95 \AA$ at phase $\phi=1.49$ in R Leo. $F_{\lambda}$ is plotted vertically, with the zero flux level for each spectrum being indicated by a tick mark on the vertical axis. Tables 5 , 7 and 8 list the maximum flux level in each line. Right: Line profiles of the forbidden transitions [Fe II] $7 \mathrm{~F}$ (4359.33 $\AA$ and $4287.40 \AA)$ and $[\mathrm{Fe} I \mathrm{II}] 21 \mathrm{~F}(4276.83 \AA$ and $4243.97 \AA)$ at phase $\phi=1.49$ in R Leo. Table 8 lists the maximum flux level in each line 
profiles are plotted against velocity rather than wavelength, because we want to analyse the atmospheric kinematics associated with pulsation. Zero velocity in the plots corresponds to the center-of-mass velocity of the star and a positive velocity indicates a motion outward from the center of the star, and visa versa. Most of the observations in the plots (left panels) were taken with a wide slit, but we also present selected observations taken with a narrow slit (right panels) to give a comparison. Clearly, since the Balmer lines have intrinsic full widths of $\sim 80 \mathrm{~km} \mathrm{~s}^{-1}$, the instrumental broadening of the wide slit $\left(15.3 \mathrm{~km} \mathrm{~s}^{-1}\right)$ does not influence the line profiles significantly.

The main result seen in the presented spectra is the change in the overall line shape with phase. The full base width (hereinafter $F B W$ ) of the hydrogen lines is around $80 \mathrm{~km} \mathrm{~s}^{-1}$ when they first appear. With increasing phase, the hydrogen lines become weaker and more narrow $\left(F B W \sim 60-70 \mathrm{~km} \mathrm{~s}^{-1}\right)$. The emission lines are generally centered around an outward velocity of $\sim 10 \mathrm{~km} \mathrm{~s}^{-1}$. Previous studies of the hydrogen lines in Miras (e.g. Fox et al. 1984) have shown similar results.

A noticeable feature of the hydrogen lines is that the strengths of superimposed absorption features diminish with advancing phase. Joy (1947) identified absorption lines of $\mathrm{TiO}$ in $\mathrm{H} \gamma$, of Fe I and $\mathrm{VI}$ in $\mathrm{H} \delta$ and of $\mathrm{TiI}$ in $\mathrm{H} \zeta$. A decrease in absorption with increasing phase is just as expected in a model where the shock producing the emission lines moves above more of the atmosphere as time progresses. The strength of the absorption features also seems to vary from star to star; for instance, superimposed absorption in $\mathrm{H} \gamma$ is stronger in $\mathrm{R} \mathrm{Aql}$ than in S Scl at comparable phases (Fig. $2 \phi=0.01$ and Fig. 4 $\phi=0.02)$. Fox et al. (1984) noted a similar variation of overlying absorption line strength from star to star and suggested that this could be due to a cycle-to-cycle variation in shock strength: at fainter maxima weaker shocks are formed and they are not able to propagate out through as much overlying absorbing material, leading to stronger absorption.

A remarkable result is the appearance of faint Balmer lines at the very early phase $\phi=0.58$ in RR Sco (Fig. 1). These emission lines are dominated by strong overlying absorption, as expected. They could also be evidence for an unusually strong shock emerging from the star in the observed pulsation cycle.

\subsection{Metal lines}

Various metal emission lines can be observed in Mira variables around maximum light and during the postmaximum phases. In the spectral range of our observation, we observed the following emission lines, which are ordered by the mutiplet number M: Si I (M 2: $4102.94 \AA)$, Mg I (M 1: $4571.10 \AA), \operatorname{MgI}(\mathrm{M} 3: 3829.32 \AA, 3832.35 \AA$ and $3838.29 \AA$ ), Fe I (M 2: $4461.65 \AA$ and $4375.93 \AA), F e I$ (M 3: $4216.18 \AA$ ), Fe I (M 42: $4307.90 \AA$ and $4202.03 \AA$ ),

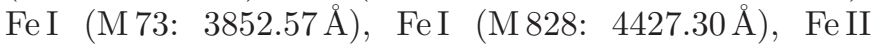

(M 38: $4583.84 \AA$ ) as well as forbidden lines [Fe II] $6 \mathrm{~F}$ $(4457.95 \AA), \quad[\mathrm{Fe} I \mathrm{II}] 7 \mathrm{~F} \quad(4359.33 \AA$ and $4287.39 \AA)$ and [Fe II $] 21 \mathrm{~F}(4276.83 \AA$ and $4243.97 \AA)$ and finially MnI (M 2: $4030.75 \AA$ ). We discuss these emission lines in the order of their appearance during the pulsation cycle.

\subsubsection{The silicon line}

The Si I line of the multiplet 2 at $4102.94 \AA$ appears at the same phases as the hydrogen lines, namely around maximum light, and varies in strength in the same way as the Balmer lines. The variation in appearance with phase is presented in Fig. 6 for RR Sco, R Aql, R Car, S Scl and $\mathrm{R}$ Hya. For comparison of wide and narrow slit spectra, additional observations, taken with a narrow slit, are shown in Fig. 7 for one phase in each of R Car and S Scl. We measured a $F B W$ of the silicon line of around $60 \mathrm{~km} \mathrm{~s}^{-1}$ when it first appears, somewhat less than the $F B W$ of the Balmer lines. As with the Balmer emission lines, the Si I emission line can be seen at the remarkably early phase $\phi=0.58$ in RR Sco (Fig. 6, left panel).

\subsubsection{Magnesium lines}

The observed MgI emission lines (M 1: 4571.10 ̊; M 3: $3829.32 \AA, 3832.35 \AA$ and $3838.29 \AA$ ) are presented in Figs. 8-10. Additional narrow slit observations are shown in the same figures for the multiplet 3 lines, and in Fig. 7 for the $4571.10 \AA$ line. Note that in some of the spectra, the $4571.10 \AA$ line appears totally in absorption.

The lines of the $\mathrm{MgI}$ multiplet 3 at $3829.32 \AA$, $3832.35 \AA$ and $3838.29 \AA$ appear around the phase of maximum when the hydrogen lines are dominant. These lines are high excitation lines (lower state excitation potential $2.7 \mathrm{eV}$ ) and the Einstein-coefficient is rather large (see Table 2). The emission lines were detected in $\mathrm{R} \mathrm{Aql}$ and R Car (Figs. 9 and 10) where we observed the early post-maximum phases in detail. We measured a $F B W$ of $\sim 30-40 \mathrm{~km} \mathrm{~s}^{-1}$. It is obvious that these lines are always blueshifted. For R Aql (Fig. 9) at phase $\phi=0.01$, the emission lines show equal velocity shifts of $\sim+10 \mathrm{~km} \mathrm{~s}^{-1}$, as can also be seen in the panel of the narrow slit observation in Fig. 9 (all emission line velocities quoted in this paper are measured at half maximum height). For R Car (Fig. 10), the velocity shift of the lines at $3829.32 \AA$ and $3832.35 \AA$ is about $+20 \mathrm{~km} \mathrm{~s}^{-1}$ when they first appear $(\phi=-0.09)$ : the weak $3838.29 \AA$ line appears to be strongly affected by overlying absorption causing it to show a smaller velocity shift of only $\sim+10 \mathrm{~km} \mathrm{~s}^{-1}$. At phase $\phi=0.11$, all multiplet 3 lines are equally blueshifted by $\sim+10 \mathrm{~km} \mathrm{~s}^{-1}$. For R Hya, we could not detect the multiplet $3 \mathrm{Mg}$ I lines at phases $\phi=0.08$ and 0.12 although one would expect them to be detectable.

At later phases, the lines of the multiplet 3 disappear and the MgI 4571.10 $\AA$ (M 1) emission line appears. The lower level of this emission line is the ground state and the Einstein-coefficient is rather low (see Table 2). Because it 
is a ground state line, it is prominent in absorption as well as emission. Before and around maximum light, two absorption components appear, especially in R Car (Fig. 10, $\phi=-0.16-0.11)$, presumably corresponding to material behind, and in front of, the emerging shock front. The majority of the observed Mg I 4571.10 $\AA$ emission lines are affected by obvious overlying absorption on the negative velocity side, although this absorption decreases as the phase advances.

The $F B W$ of the $\mathrm{MgI} 4571.10 \AA$ emission line is generally around $40-50 \mathrm{~km} \mathrm{~s}^{-1}$ when it first appears and it is blueshifted by $\sim+10 \mathrm{~km} \mathrm{~s}^{-1}$ with respect to the center-ofmass velocity. There is a general decrease in the blueshift towards minimum light; this is probably due to a combination of a real slowing of the shock and a decrease in overlying absorption on the red edge. In R Aql (Fig. 9), the velocity shift around the minimum phase $(\phi=0.50)$ seems to increase again, possibly due to some asymmetry in the upper atmosphere being transversed by the shock.

$\mathrm{R}$ Leo is an interesting case to study, since there are two minima observed which appear rather different. Around the first minimum, the $\mathrm{MgI} 4571 \AA$ line was very faint and narrow $\left(F B W \sim 30 \mathrm{~km} \mathrm{~s}^{-1}\right)$ with the velocity shift decreasing from $+5 \mathrm{~km} \mathrm{~s}^{-1}$ to $+2 \mathrm{~km} \mathrm{~s}^{-1}$ for the phases $\phi=0.43$ to $\phi=0.63$. At the next minimum, the emission line was bright and showed a $F B W$ of $\sim 40 \mathrm{~km} \mathrm{~s}^{-1}$ and the line was centered at a velocity shift of $\sim+3 \mathrm{~km} \mathrm{~s}^{-1}$. The AAVSO light curve shows that the light maximum preceding the first minimum was much fainter than the maximum preceding the second minimum. A bright preceding maximum obviously leads to a much wider and stronger Mg I $4571.10 \AA$ emission line. This is consistent with the hypothesis that a stronger (visually brighter) maximum is associated with a stronger shock.

The development of the $\mathrm{MgI} 4571 \AA$ line in R Hya (Fig. 8, right panel), the star with the longest period (389 d) of our sample, is obviously different. The emission line appears at a relatively early phase $(\phi=0.12)$ and it has a sharp low velocity edge indicative of strong overlying absorption. Clearly, velocities derived from this line need to be treated with caution, at least until very advanced phases.

\subsubsection{Neutral iron lines}

Out of the variety of neutral iron emission lines in Mira variables, we studied the following: (M 2) $4461.65 \AA$ and $4375.93 \AA$, (M 3) 4216.18

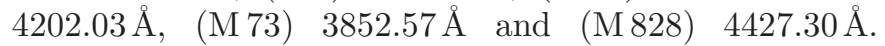
Figs. 11-16 show the observed FeI lines for all stars of our sample. For S Scl, R Hya and R Aql we present narrow slit as well as broad slit observations for some of the neutral iron lines.

It is obvious that several of the FeI emission lines, namely FeI $4307.90 \AA, 4202.03 \AA$ and $3852.57 \AA$, appear brightly during the post-maximum phase of every star of our sample. Table 2 shows that these are all high exci- tation lines (lower level excitation potential 1.5-2.2 eV) with large Einstein-coefficents. It has been suggested that the great strength of all three lines is the result of fluorescence, the lines being pumped by the Mg II 2795, $2802 \AA$ lines (Thackeray 1937; Willson 1976).

The $3852.57 \AA$ line appears around maximum visible light with a very narrow $F B W$ of $\sim 25-30 \mathrm{~km} \mathrm{~s}^{-1}$. At later phases it widens up to a $F B W$ of $\sim 40-60 \mathrm{~km} \mathrm{~s}^{-1}$. It appears that this is due to overlying absorption at early phases removing the negative velocity side of the line (see R Car, R Hya and R Aql in Figs. 12, 14 and 15).

The $4202.03 \AA$ line also appears near maximum light (see R Car, S Scl and R Aql in Figs. 12, 13 and 15). At early phases, it shows an obvious inverted P-Cygni profile caused by overlying absorption of infalling material.

The $4307.90 \AA$ line does not appear as prominently as the previous line for phases $\lesssim 0.2$ even though both lines have identical upper states (see Table 2). This is almost certainly due to more overlying absorption affecting this line: around maximum light in R Car, S Scl and R Aql (Figs. 12, 13 and 15), the line is totally in absorption while the $4202.03 \AA$ line shows distinct emission. Multiple absorption components are clearly visible cutting into the emission line except at the latest phases. This may be partly self-absorption by pre- and post-shock material as the $4307.90 \AA$ has the largest Einstein-coefficent of the two lines. The large Einstein-coefficent may also explain the fact that the $4307.90 \AA$ line persists the longest and has the largest flux (see Table 6) of the two lines.

All other Fe I emission lines observed are low excitation lines (see Table 2). The $4375.93 \AA$ line of the multiplet 2 appears around the phase of maximum light (see RR Sco, R Car and R Leo in Figs. 11, 12, 16), which is an exceptional early appearance for a low excitation line. The line shows an obvious inverted P Cygni profile with a steep red wing: the absorption component decreases towards later phases. The $4461.65 \AA$ line was only detected in emission in R Car at the phase $\phi=0.39$ and in R Leo at $\phi=1.49$ (Figs. 12 and 16). Although it belongs to the same multiplet (2) as the $4375.93 \AA$ line, it is not as prominent at early phases as the latter.

The remainder of the observed Fe I low excitation lines only appear in some stars and no regularity can be seen. The Fe I $4427 \AA$ emission line was observed in RR Sco and R Leo at just one phase each (see Figs. 11 and 16). Finally, the low excitation line at $4216.18 \AA$ was observed in R Leo at the phase $\phi=1.49$.

\subsubsection{Ionized iron lines}

The permitted Fe II $4583.84 \AA$ emission line was observed in three of the stars of our sample, namely RR Sco, R Car and R Leo (Figs. 17 and 18). This is a high excitation line (lower level excitation potential $2.8 \mathrm{eV}$ ) which appears towards the minimum light of the star, at phase $\phi=0.29$ in RR Sco, $\phi=0.39$ in R Car and $\phi=1.49$ in R Leo. The $F B W$ varies from $\sim 35 \mathrm{~km} \mathrm{~s}^{-1}$ in RR Sco and R Car to 
$\sim 45 \mathrm{~km} \mathrm{~s}^{-1}$ in $\mathrm{R}$ Leo. The velocity shifts of the Fe II line are around $+2 \mathrm{~km} \mathrm{~s}^{-1}$ in these stars.

In R Leo and R Car, forbidden [FeII] lines can be clearly seen at the same phase as the permitted FeII line at $4583.84 \AA$ (Figs. 17 and 18). In R Leo at phase $\phi=1.49$, following the bright maximum, we found the transitions [Fe II] $6 \mathrm{~F} \quad(4457.95 \AA), \quad[\mathrm{Fe} \mathrm{II}] 21 \mathrm{~F} \quad(4276.83 \AA$ and $4243.97 \AA)$ and [Fe II] $7 \mathrm{~F}(4359.33 \AA$ and $4287.39 \AA)$. No forbidden lines could be seen in the previous cycle following a fainter maximum: the cycle-to-cycle variability of the forbidden lines is consistent with the suggestion noted earlier that stronger shocks are associated with brighter maxima.

In R Car at phase $\phi=0.39$, we identified only the two [Fe II] 7F emission lines at $4359.33 \AA$ and $4287.39 \AA$. Unfortunately, the observation for R Car at phase $\phi=0.39$ was taken with a narrow slit in poor weather conditions, so the signal to noise of these faint lines is low. Other forbidden lines were probably present but undetectable. In RR Sco we could not identify any of the forbidden lines, although we could clearly detect the permitted Fe II line (Fig. 17).

The $F B W$ of the [FeII] 7F emission lines in R Car is $\sim 21-26 \mathrm{~km} \mathrm{~s}^{-1}$. The velocity shift was measured at $0-2 \mathrm{~km} \mathrm{~s}^{-1}$, which is the same as for the permitted Fe II $4583.84 \AA$ emission. In R Leo, all the [Fe II] emission lines have a $F B W$ of $\sim 40 \mathrm{~km} \mathrm{~s}^{-1}$. The velocity shifts are approximately $0-2 \mathrm{~km} \mathrm{~s}^{-1}$ for the $7 \mathrm{~F}$ and the $21 \mathrm{~F}$ emission lines, similar to the velocity shift of permitted Fe II $4583.84 \AA$ line.

\subsubsection{Manganese line}

The manganese line at $4030.75 \AA$ is a ground state line. It was observed in just one star, namely $\mathrm{R}$ Leo, at phase $\phi=1.49$ (Fig. 18). The Mn I emission line shows a $F B W$ of $\sim 28 \mathrm{~km} \mathrm{~s}^{-1}$ and has a large velocity shift of $10 \mathrm{~km} \mathrm{~s}^{-1}$, unlike the Fe II and [Fe II] lines at the same phase. As with the neutral Fe lines of low excitation (e.g. Fe I $4375.93 \AA$ ), the sharper red edge of the line indicates absorption by infalling, neutral Mn atoms in the layers above the shock.

\section{Line fluxes}

Total line fluxes $F_{\text {tot }}\left(\mathrm{erg} \mathrm{cm}^{-2} \mathrm{~s}^{-1}\right)$ for the observed emission lines are given in Tables 4 to 8 , along with the peak flux $F_{\text {peak }}\left(\mathrm{erg} \mathrm{cm}^{-2} \mathrm{~s}^{-1} \AA^{-1}\right)$ in the line plots. The peak flux can be used in conjunction with Figs. 1-18 (except for the narrow slit observations) to get intensities through the line profiles. Note that no correction for superimposed absorption lines has been made in calculating total line fluxes.

Figures 19-22 show the variation with phase of all fluxes measured in each star of our sample. For the Balmer lines, both the data given in this paper and the data of Fox et al. (1984) are used in the plots. The typical variation with phase can be seen best in R Car (Fig. 22), for which a separate plot is shown for the Balmer lines. The
Balmer lines $\mathrm{H} \gamma$ and $\mathrm{H} \delta$ appear first, roughly between phases -0.4 and -0.2 (see the Fox et al. 1984 data for R Car, S Scl, RR Sco and R Aql as well as the present data for R Car - Figs. 20, 21 and 22). The lines then strengthen rapidly, reaching maximum flux around phase -0.1 . At this phase, the other Balmer lines are observed too although they tend to reach maximum flux around phase +0.1 when the amount of overlying absorption is reduced. The Balmer lines finally vanish about phase $\phi=0.4$.

Fox et al. (1984) noted that the Balmer line fluxes can vary enormously from cycle to cycle (see $o$ Ceti in their data), with brighter maxima yielding brighter Balmer lines. A comparison of their fluxes with ours for R Car also shows this effect. The Balmer lines we observed in R Car are roughly ten times brighter than the lines observed by Fox et al: at the same time, the AAVSO light curves show that the visual maximum during our observations $(V \sim 3.8)$ was about one magnitude brighter than the maximum $(V \sim 4.8)$ during which Fox et al. made their observations.

The Si I $4102 \AA$ (M 2) high excitation line appears in all stars near maximum light. The line flux is always less than in the nearby Balmer line $\mathrm{H} \delta$. In general, the variation with phase and the phase of the peak flux is similar to that of the hydrogen lines, although the peak flux is a factor of $\sim 10$ less than in the hydrogen lines.

The high excitation lines of multiplet 3 of $\mathrm{MgI}$ (3829.32 $\AA$, 3832.35 $\mathrm{A}$ and $3838.29 \AA)$ appear near maximum light and were observed in R Aql and R Car (Fig. 21 right panel, and Fig. 22 left panel). The flux in these lines is about a factor of 30 less than in the Balmer lines. The lines disappear at $\phi \sim 0.2-0.3$ and are replaced by the low excitation line of MgI (M1) at 4571.10 $\AA$. The latter appears around $\phi \sim 0.2-0.3$ and was observed in every star of our sample, disappearing finally at the late phase $\phi \sim 0.6$. It appears exceptionally early $(\phi=0.12)$ in $\mathrm{RHya}$, as already noted in Sect. 3.2.2.

The FeI lines show a rather complex variation with phase. In general, one can divide them into two groups: type A, those appearing early in the cycle ( $\sim$ maximum); and type B, those appearing around $\phi \sim 0.3-0.4$. The first group consists of $4202.03 \AA$ (M 42), 3852.57 $($ M 73) (both high excitation lines) and the $4375.93 \AA$ (M 2) (a low excitation line). Variation of flux with phase in R Hya, R Aql and R Car can be seen Fig. 19 right panel, Fig. 21 right panel, and Fig. 22 left panel). These lines vary less rapidly than the Balmer or Si I lines and reach their maximum at the later phase of $\phi \sim 0.1$.

The type B FeI emission lines 4427.29 $\AA$ (M 828), $4461.65 \AA$ (M 2), $4216.18 \AA$ (M 3) and $4307.90 \AA$ (M 42) appear around $\phi \sim 0.3-0.4$ and are usually fainter than Fe I emission lines discussed above. Apart from the $4307.90 \AA$ line, these emission lines are all of low excitation. As noted in Sect. 3.2.3, the $4307.90 \AA$ line has the same upper level as the $4202.03 \AA$ line of the same multiplet and its late appearance and low flux relative to the $4202.03 \AA$ line is almost certainly due to overlying absorption. However, the flux in the $4307.90 \AA$ line at late phases is usually 
Table 4. H and Si I line fluxes

\begin{tabular}{|c|c|c|c|c|c|c|c|c|c|c|c|}
\hline \multirow[b]{2}{*}{ Star } & \multirow[b]{2}{*}{ Phase } & \multicolumn{2}{|c|}{$\mathbf{H}_{\gamma}(4340 \AA)$} & \multicolumn{2}{|c|}{$\mathbf{H}_{\delta}(4101 \AA)$} & \multicolumn{2}{|c|}{$\mathbf{H}_{\zeta}(3889 \AA)$} & \multicolumn{2}{|c|}{$\mathbf{H}_{\eta}(3835 \AA)$} & \multicolumn{2}{|c|}{ Si I $($ M $2 ; 4102 \AA)$} \\
\hline & & $F_{\text {peak }}$ & $F_{\text {tot }}$ & $F_{\text {peak }}$ & $F_{\text {tot }}$ & $F_{\text {peak }}$ & $F_{\text {tot }}$ & $F_{\text {peak }}$ & $F_{\text {tot }}$ & $F_{\text {peak }}$ & $F_{\text {tot }}$ \\
\hline \multirow[t]{2}{*}{ RR Sco } & 0.29 & $8.2-12$ & $3.2-12$ & $1.1-11$ & $3.7-12$ & $5.3-12$ & $1.6-12$ & $4.3-12$ & $1.5-12$ & $8.5-13$ & $7.2-13$ \\
\hline & 0.58 & $5.0-13$ & $2.7-13$ & $3.5-12$ & $1.2-12$ & - & - & - & - & $1.2-12$ & $6.8-13$ \\
\hline \multirow[t]{3}{*}{$\mathrm{R}$ Aql } & 0.01 & $1.4-10$ & $8.3-11$ & $3.5-10$ & $1.6-10$ & $1.2-10$ & $5.8-11$ & $1.0-10$ & $5.2-11$ & $1.9-11$ & $1.1-11$ \\
\hline & 0.32 & $3.0-12$ & $1.6-12$ & $3.2-12$ & $1.0-12$ & $1.1-12$ & $4.9-13$ & $1.2-12$ & $2.8-13$ & - & - \\
\hline & 0.39 & $1.5-12$ : & $1.0-12:$ & 1.9-12: & $6.2-13:$ & - & - & - & - & - & - \\
\hline \multirow{4}{*}{ R Car } & -0.16 & 6.1-11: & 2.0-11: & 1.6-10: & $4.7-11:$ & - & - & - & - & 1.9-11: & $6.9-12$ : \\
\hline & -0.09 & $1.1-9:$ & $7.8-10:$ & 1.8-9: & $9.7-10$ : & $3.1-10:$ & 1.9-10: & 1.9-10: & $1.2-10:$ & $1.2-10$ : & $5.2-11:$ \\
\hline & 0.11 & $6.8-10$ & $2.7-10$ & $1.0-9$ & $3.7-10$ & $5.0-10$ & $1.9-10$ & $4.1-10$ & $1.6-10$ & $4.3-11$ & $1.2-11$ \\
\hline & 0.39 & 3.7-12: & $2.2-12$ : & - & - & - & - & - & - & - & - \\
\hline $\mathrm{S} \mathrm{Scl}$ & 0.02 & $7.0-11$ & $3.7-11$ & $3.3-10$ & $1.4-10$ & $8.0-11$ & $4.1-11$ & $5.0-11$ & $3.5-11$ & $1.4-11$ & $6.8-12$ \\
\hline \multirow[t]{3}{*}{ R Hya } & 0.08 & 3.0-10: & 1.3-10: & 2.7-10: & 1.0-10: & 1.7-10: & 6.0-11: & 8.8-11: & 3.0-11: & 1.8-11: & $6.8-12$ \\
\hline & 0.12 & 2.1-10: & 9.1-11: & 1.1-10: & 4.3-11: & 9.5-11: & 3.4-11: & $3.2-11$ : & 1.0-11: & $2.2-11$ : & 4.9-12: \\
\hline & 0.21 & $3.2-11$ & $1.7-11$ & $2.2-11$ & $8.5-12$ & $1.3-11$ & $4.3-12$ & $1.2-11$ & $5.4-12$ & $2.4-12$ & $9.8-13$ \\
\hline
\end{tabular}

Notes: $F_{\text {peak }}$ is the peak flux in the line in erg cm ${ }^{-2} \mathrm{~s}^{-1} \AA^{-1} . F_{\text {tot }}$ is the total flux in the line in $\mathrm{erg} \mathrm{cm}^{-2} \mathrm{~s}^{-1}$.

Numbers followed by a colon correspond to fluxes derived by normalizing to the AAVSO light curve (see text for details).

Table 5. Mg I and Mn I line fluxes

\begin{tabular}{|c|c|c|c|c|c|c|c|c|c|c|c|}
\hline \multirow[b]{2}{*}{ Star } & \multirow[b]{2}{*}{ Phase } & \multicolumn{2}{|c|}{$\mathbf{M g} \mathbf{I}(\mathrm{M} 1 ; 4571 \AA)$} & \multicolumn{2}{|c|}{ Mg I (M 3; $3829 \AA$ ) } & \multicolumn{2}{|c|}{ Mg I (M 3; $3832 \AA)$} & \multicolumn{2}{|c|}{$\mathbf{M g} \mathbf{I}(\mathrm{M} 3 ; 3838 \AA)$} & \multicolumn{2}{|c|}{ Mn I $($ M $2 ; 4030 \AA)$} \\
\hline & & $F_{\text {peak }}$ & $F_{\text {tot }}$ & $F_{\text {peak }}$ & $F_{\text {tot }}$ & $F_{\text {peak }}$ & $F_{\text {tot }}$ & $F_{\text {peak }}$ & $F_{\text {tot }}$ & $F_{\text {peak }}$ & $F_{\text {tot }}$ \\
\hline \multirow[t]{2}{*}{ RR Sco } & 0.29 & $1.3-12$ & $4.5-13$ & - & - & - & - & - & - & - & - \\
\hline & 0.48 & $2.9-13$ & $6.6-14$ & - & - & - & - & - & - & - & - \\
\hline \multirow[t]{4}{*}{ R Aql } & 0.01 & - & - & $1.1-11$ & $2.8-12$ & $1.4-11$ & $3.4-12$ & $1.1-11$ & $2.8-12$ & - & - \\
\hline & 0.32 & $2.0-12$ & $4.8-13$ & - & - & - & - & - & - & - & - \\
\hline & 0.39 & 2.6-12: & $7.5-13:$ & - & - & - & - & - & - & - & - \\
\hline & 0.50 & $6.5-13$ & $1.7-13$ & - & - & - & - & - & - & - & - \\
\hline \multirow[t]{6}{*}{ R Car } & -0.09 & - & - & 3.6-11: & 1.0-11: & $3.4-11:$ & $5.7-12:$ & 1.9-11: & $3.7-12$ : & - & - \\
\hline & 0.11 & - & - & $2.7-11$ & $9.6-12$ & $3.2-11$ & $7.2-12$ & $3.0-11$ & $1.5-12$ & - & - \\
\hline & 0.39 & 1.8-11: & $3.7-12:$ & - & - & - & - & - & - & - & - \\
\hline & 0.45 & 6.7-12: & 1.5-12: & - & - & - & - & - & - & - & - \\
\hline & 0.57 & $1.0-12$ & $3.1-13$ & - & - & - & - & - & - & - & - \\
\hline & 0.66 & $4.0-13$ & $1.4-13$ & - & - & - & - & - & - & - & - \\
\hline \multirow[t]{3}{*}{ R Leo } & 0.43 & 6.6-12: & 1.4-12: & - & - & - & - & - & - & - & - \\
\hline & 0.63 & $9.5-13$ & $1.8-13$ & - & - & - & - & - & - & - & - \\
\hline & 1.49 & $7.4-12$ & $2.0-12$ & - & - & - & - & - & - & $8.8-13$ & $2.6-13$ \\
\hline \multirow[t]{2}{*}{ S Scl } & 0.52 & $2.6-13$ & $6.8-14$ & - & - & - & - & - & - & - & - \\
\hline & 0.58 & $1.5-13$ & $4.9-14$ & - & - & - & - & - & - & - & - \\
\hline \multirow[t]{3}{*}{ R Hya } & 0.12 & $9.4-12$ : & $3.2-12$ : & - & - & - & - & - & - & - & - \\
\hline & 0.21 & $8.4-12$ & $3.1-12$ & - & - & - & - & - & - & - & - \\
\hline & 0.53 & 1.4-12: & 4.7-13: & - & - & - & - & - & - & - & - \\
\hline
\end{tabular}

Notes: Same as Table 4.

greater than in other late emission lines of Fe I $4427.29 \AA$, $4461.65 \AA$ and $4216.18 \AA$. Its flux and variation with phase is quite similar to the $\mathrm{Mg}$ I $4571.10 \AA$ line.

Fe II lines were observed at late phases in a number of stars. Line fluxes of the permitted Fe II $4583.84 \AA$ (M 38) emission line are shown for RR Sco, R Car and R Leo in Figs. 21, 22 and 19 (left panels). We note that this high excitation line appears around $\phi \sim 0.3-0.5$ and is one of the faintest lines observed. Fluxes for the forbidden lines [Fe II] $7 \mathrm{~F}$ at $4359.33 \AA$ and $4287.40 \AA$ are plotted in Fig. 22 (left panel) for R Car at phase $\phi=0.39$ and in Fig. 19 (left panel) for R Leo at $\phi=1.49$. The line flux in R Car is similar to that of the permitted Fe II emission line at $4583.84 \AA$. In $\mathrm{R}$ Leo, however, the forbidden line flux is $\sim 3$ times higher than the permitted line flux, at least for $\phi=1.49$ where the forbidden lines were observed. At phase $\phi=1.49$ in R Leo, we also measured the line fluxes of the $[\mathrm{Fe} \mathrm{II}] 6 \mathrm{~F}$ and $[\mathrm{Fe} \mathrm{II}] 21 \mathrm{~F}$ emission lines. These lines are weaker than the $[\mathrm{Fe} \mathrm{II}] 7 \mathrm{~F}$ lines: their flux is comparable to the faint, late-appearing Fe I lines.

It is worth noting the much higher metal emission line fluxes during the second observed minimum of R Leo. Comparing the line fluxes of MgI $4571.10 \AA$ and Fe I $4307.90 \AA$, we see that the MgI line flux is stronger 
Table 6. Fe I line fluxes

\begin{tabular}{|c|c|c|c|c|c|c|c|c|c|c|c|}
\hline \multirow[b]{2}{*}{ Star } & \multirow[b]{2}{*}{ Phase } & \multicolumn{2}{|c|}{$\mathbf{F e} \mathbf{I}(\mathrm{M} 2 ; 4461 \AA)$} & \multicolumn{2}{|c|}{ Fe I $(M 2 ; 4375 \AA)$} & \multicolumn{2}{|c|}{ Fe I $(M 3 ; 4216 \AA)$} & \multicolumn{2}{|c|}{ Fe I $(\mathrm{M} 42 ; 4307 \AA)$} & \multicolumn{2}{|c|}{$\mathbf{F e} \mathbf{I}(\mathrm{M} 42 ; 4202 \AA)$} \\
\hline & & $F_{\text {peak }}$ & $F_{\text {tot }}$ & $F_{\text {peak }}$ & $F_{\text {tot }}$ & $F_{\text {peak }}$ & $F_{\text {tot }}$ & $F_{\text {peak }}$ & $F_{\text {tot }}$ & $F_{\text {peak }}$ & $F_{\text {tot }}$ \\
\hline \multirow[t]{2}{*}{ RR Sco } & 0.29 & - & - & $3.6-13$ & $1.2-13$ & - & - & $1.4-12$ & $7.1-13$ & $3.9-12$ & $7.4-13$ \\
\hline & 0.48 & - & - & - & - & - & - & $1.3-13$ & $4.7-14$ & - & - \\
\hline \multirow{4}{*}{ R Aql } & 0.01 & - & - & - & - & - & - & - & - & $9.9-12$ & $2.3-12$ \\
\hline & 0.32 & - & - & - & - & - & - & $9.7-13$ & $5.0-13$ & $2.2-12$ & $5.1-13$ \\
\hline & 0.39 & - & - & - & - & - & - & 1.4-12: & $5.6-13:$ & $2.0-12:$ & 4.3-13: \\
\hline & 0.50 & - & - & - & - & - & - & $3.5-13$ & $1.1-13$ & - & - \\
\hline \multirow[t]{6}{*}{ R Car } & -0.09 & - & - & 1.6-11: & 1.6-12: & - & - & - & - & $2.9-11:$ & $6.5-12:$ \\
\hline & 0.11 & - & - & $2.4-11$ & $4.4-12$ & - & - & - & - & $4.5-11$ & $1.0-11$ \\
\hline & 0.39 & 1.6-12: & $2.7-13:$ & 1.4-12: & 1.7-13: & - & - & 1.2-11: & 5.6-12: & $9.5-12$ : & $3.5-12:$ \\
\hline & 0.45 & - & - & - & - & - & - & $2.1-12$ : & 7.6-13: & $1.8-12$ : & 6.2-13: \\
\hline & 0.57 & - & - & - & - & - & - & $7.3-13$ & $2.1-13$ & - & - \\
\hline & 0.66 & - & - & - & - & - & - & $2.6-13$ & $6.6-14$ & - & - \\
\hline \multirow[t]{3}{*}{ R Leo } & 0.43 & - & - & - & - & - & - & 1.5-12: & 2.8-13: & - & - \\
\hline & 0.63 & - & - & - & - & - & - & $4.1-13$ & $1.0-13$ & - & - \\
\hline & 1.49 & $3.6-13$ & $7.9-14$ & $4.0-13$ & $8.4-14$ & $2.5-13$ & $5.3-14$ & $3.7-12$ & $1.5-12$ & $2.6-12$ & $1.0-12$ \\
\hline \multirow[t]{3}{*}{$\mathrm{S} \mathrm{Scl}$} & 0.02 & - & - & - & - & - & - & - & - & $6.5-12$ & $1.7-12$ \\
\hline & 0.52 & - & - & - & - & - & - & $2.6-13$ & $8.4-14$ & - & - \\
\hline & 0.58 & - & - & - & - & - & - & $1.3-13$ & $3.9-14$ & - & - \\
\hline \multirow[t]{4}{*}{ R Hya } & 0.08 & - & - & - & - & - & - & $8.0-12:$ & $3.1-12:$ & 4.3-11: & 1.1-11: \\
\hline & 0.12 & - & - & - & - & - & - & $9.8-12:$ & 2.8-12: & 1.9-11: & $5.2-12:$ \\
\hline & 0.21 & - & - & - & - & - & - & $5.5-12$ & $2.5-12$ & $1.1-11$ & $2.9-12$ \\
\hline & 0.53 & - & - & - & - & - & - & $2.5-12:$ & 7.8-13: & - & - \\
\hline
\end{tabular}

Notes: Same as Table 4.

Table 7. FeI and Fe II line fluxes

\begin{tabular}{lrllllll}
\hline & \multicolumn{3}{c}{ Fe I $(\mathrm{M} 73 ; 3852 \AA)$} & \multicolumn{2}{l}{ Fe I $(\mathrm{M} 828 ; 4427 \AA)$} & \multicolumn{2}{l}{ Fe II $(\mathrm{M} 38 ; 4583 \AA)$} \\
Star & Phase & $F_{\text {peak }}$ & $F_{\text {tot }}$ & $F_{\text {peak }}$ & $F_{\text {tot }}$ & $F_{\text {peak }}$ & $F_{\text {tot }}$ \\
\hline RR Sco & 0.29 & $4.8-12$ & $1.2-12$ & $4.2-13$ & $9.2-14$ & $2.3-13$ & $4.7-14$ \\
R Aql & 0.01 & $1.5-11$ & $3.5-12$ & - & - & - & - \\
& 0.32 & $3.2-12$ & $9.7-13$ & - & - & - & - \\
R Car & -0.09 & $2.7-11:$ & $3.9-12:$ & - & - & - & - \\
& 0.11 & $1.1-10$ & $2.9-11$ & - & - & - & - \\
& 0.39 & - & - & - & - & $7.7-13:$ & $5.8-13:$ \\
R Leo & 1.49 & - & - & $4.2-13$ & $9.1-14$ & $2.4-13$ & $7.3-14$ \\
S Scl & 0.02 & $8.5-12$ & $1.9-12$ & - & - & - & - \\
R Hya & 0.08 & $3.6-11:$ & $6.8-12:$ & - & - & - & - \\
& 0.12 & $1.9-11:$ & $4.2-12:$ & - & - & - & - \\
& 0.21 & $8.3-12$ & $3.7-12$ & - & - & - & - \\
\hline
\end{tabular}

Notes: Same as Table 4.

Table 8. [Fe II] line fluxes

\begin{tabular}{|c|c|c|c|c|c|c|c|c|c|c|c|}
\hline \multirow[b]{2}{*}{ Star } & \multirow[b]{2}{*}{ Phase } & \multicolumn{2}{|c|}{$\begin{array}{l}\text { [FeII] 6F } \\
(4457 \AA)\end{array}$} & \multicolumn{2}{|c|}{$\begin{array}{l}{[\mathbf{F e I I}] \mathbf{7 F}} \\
(4359 \AA)\end{array}$} & \multicolumn{2}{|c|}{$\begin{array}{l}{[\mathbf{F e I I}] \mathbf{7 F}} \\
(4287 \AA)\end{array}$} & \multicolumn{2}{|c|}{$\begin{array}{l}{[\mathbf{F e I I}] \mathbf{2 1 F}} \\
(4276 \AA)\end{array}$} & \multicolumn{2}{|c|}{$\begin{array}{l}{[\mathbf{F e I I}] \mathbf{2 1 F}} \\
(4243 \AA)\end{array}$} \\
\hline & & $F_{\text {peak }}$ & $F_{\text {tot }}$ & $F_{\text {peak }}$ & $F_{\text {tot }}$ & $F_{\text {peak }}$ & $F_{\text {tot }}$ & $F_{\text {peak }}$ & $F_{\text {tot }}$ & $F_{\text {peak }}$ & $F_{\text {tot }}$ \\
\hline R Car & 0.39 & - & - & $1.2-12$ : & $2.5-13:$ & $2.7-12$ : & 5.5-13: & 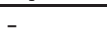 & - & - & - \\
\hline R Leo & 1.49 & $2.3-13$ & $9.0-14$ & $5.4-13$ & $1.4-13$ & $7.8-13$ & $3.0-13$ & $2.6-13$ & $7.9-14$ & $5.4-13$ & $1.4-13$ \\
\hline
\end{tabular}

Notes: Same as Table 4 for [FeII] line flux 

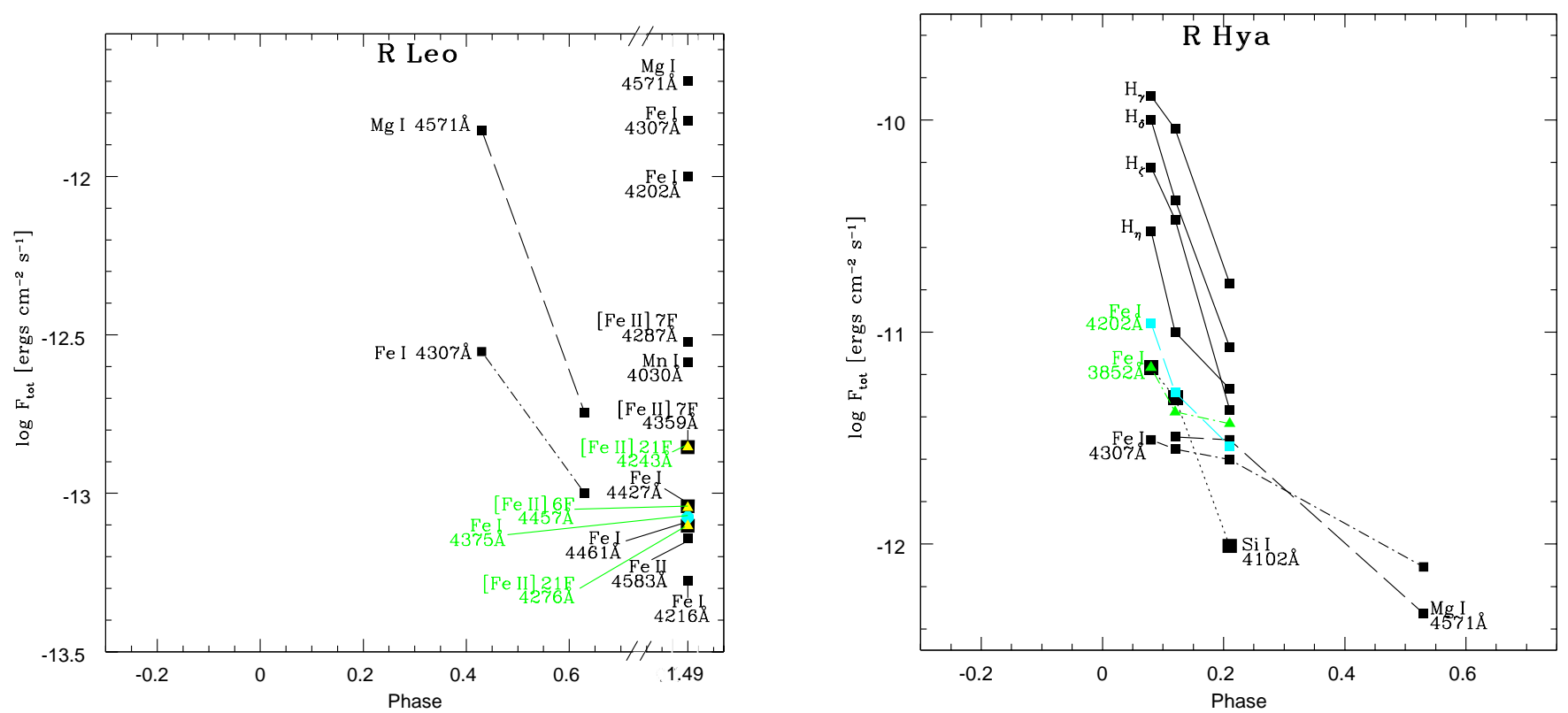

Fig. 19. Left: Absolute line fluxes plotted against phase in R Leo. Note the change in scale of the phase for we plotted the fluxes of the minimum of the next cycle in the same panel. Note also that several line fluxes are coincident at phase $\phi=1.49$ ([Fe II] $7 \mathrm{~F}$ $4359 \AA$ and [Fe II] $21 \mathrm{~F} 4243 \AA$; Fe I $4427 \AA$ and [Fe II] $6 \mathrm{~F} 4457 \AA$; Fe I $4461 \AA$, Fe I $4375 \AA$ and [Fe II] 21F $4276 \AA$ ). Right: Absolute line fluxes plotted against phase in R Hya. Note the coincident line fluxes at phases $\phi=0.08$ ( $\mathrm{SiI}$ and FeI $3852 \AA$ ) as well as $\phi=0.12(\mathrm{Si}$ I and Fe I $4202 \AA)$

by a factor of $\sim 3$ in the second cycle whereas the Fe I line flux is stronger by a factor of almost 10. Finally, in Fig. 23 we summarize the overall duration and flux variation with phase of emission lines in M-type Miras.

\section{Line velocities}

Another property of the emission line spectra that is useful for comparison with theoretical models of Mira atmospheres is the velocity of the emission lines. This line velocity should represent the velocity of the emission region associated with the shock wave passing through the Mira atmosphere.

In Fig. 24, we plot the velocities (relative the stellar center-of-mass) of representative emission lines against the phase of the pulsation cycle. The velocities are defined at half-height of the line profiles and are obtained from all stars in the sample. When overlying absorption is dominant (for example, at early phases of the $\mathrm{H} \delta$ line), no velocity measurement was made.

The plot clearly shows that when the shock emerges from deep in the photosphere, the post-shock emission region has a measured outward velocity of $\sim 10-12 \mathrm{~km} \mathrm{~s}^{-1}$. This result is in good agreement with the velocity measured in the infrared (Hinkle 1978; Hinkle et al. 1982, 1984) for deep pulsating layers when converted to centerof-mass velocities (Wood 1987). As phase advances, the emission line velocity decreases until it becomes essentially zero around minimum light. Note that the material whose velocity is measured by the emission lines is associated with the near-shock zone: this is quite different from the

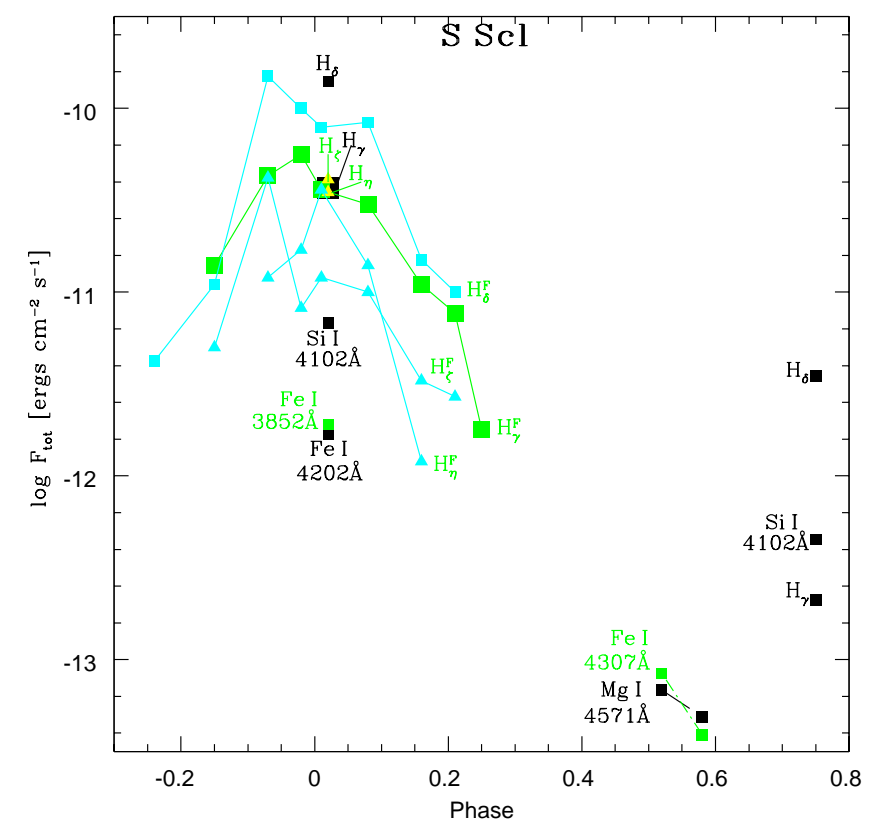

Fig. 20. Absolute line fluxes plotted against phase in S Scl. Note the nearly equal fluxes for $\mathrm{H}_{\zeta}, \mathrm{H}_{\gamma}$ and $\mathrm{H}_{\eta}$ at phase $\phi=$ 0.02 . At phase $\phi=0.75$ the fluxes of newly appearing emission lines are shown. The Balmer line fluxes $\mathrm{H}_{\gamma}^{F}, \mathrm{H}_{\delta}^{F}, \mathrm{H}_{\zeta}^{F}$ and $\mathrm{H}_{\eta}^{F}$ from Fox et al. (1984) are plotted in grey. At $\phi=-0.07 \mathrm{H}_{\gamma}^{F}$ and $\mathrm{H}_{\zeta}^{F}$ are coincident while $\mathrm{H}_{\gamma}^{F}$ and $\mathrm{H}_{\eta}^{F}$ are equal at $\phi=0.0$ and also nearly coincide with $\mathrm{H}_{\eta}$

deeper, infall zone whose velocity is measured near minimum light by the infrared spectra.

At first sight, it is surprising that in the average Mira the apparent velocity of the emission lines approaches zero 

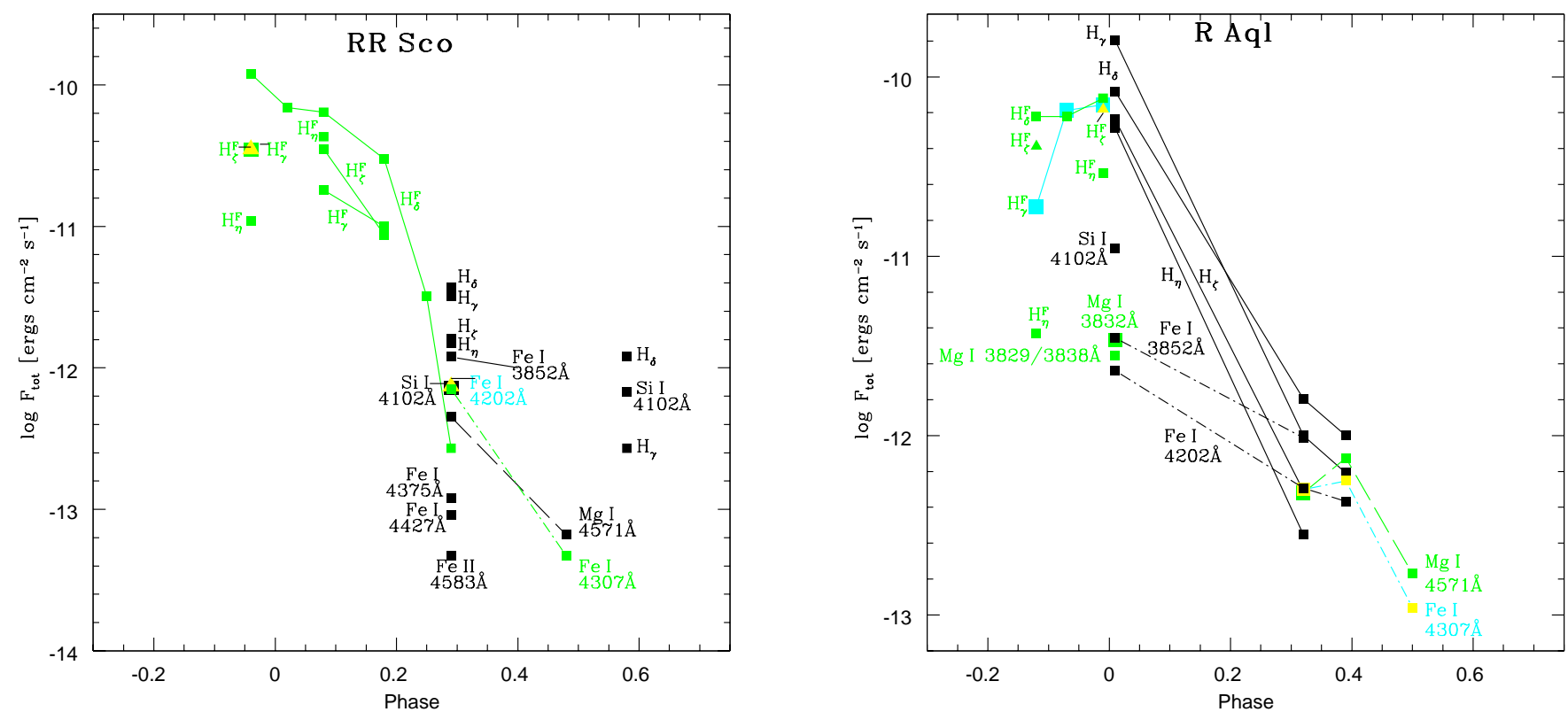

Fig. 21. Left: Absolute line fluxes plotted against phase in RR Sco. Note that at phase $\phi=0.29$ the fluxes of Si I $4102 \AA$, Fe I $4202 \AA$ and Fe I $4307 \AA$ are nearly equal. At phase $\phi=0.58$ the fluxes of newly appearing emission lines are shown. Balmer line fluxes measured by Fox et al. (1984) are plotted in grey and marked with $\mathrm{H}_{\gamma}^{F}, \mathrm{H}_{\delta}^{F}, \mathrm{H}_{\zeta}^{F}$ and $\mathrm{H}_{\eta}^{F}$ (fluxes for $\mathrm{H}_{\gamma}^{F}$ and $\mathrm{H}_{\zeta}^{F}$ are coincident at $\phi=-0.04)$. Right: Absolute line fluxes plotted against phase in R Aql. Note that there are coinciding points at phase $\phi=0.01(\mathrm{Mg}$ I $3832 \AA$ and Fe I $3852 \AA)$ as well as $\phi=0.32\left(\mathrm{H}_{\zeta}\right.$, Fe I $4307 \AA$ and $\left.\mathrm{Mg} \mathrm{I} \AA\right)$. The Balmer line fluxes from Fox et al. (1984) are plotted in grey (fluxes for $\mathrm{H}_{\gamma}^{F}, \mathrm{H}_{\delta}^{F}, \mathrm{H}_{\zeta}^{F}$ are nearly equal at $\phi=-0.01$ )
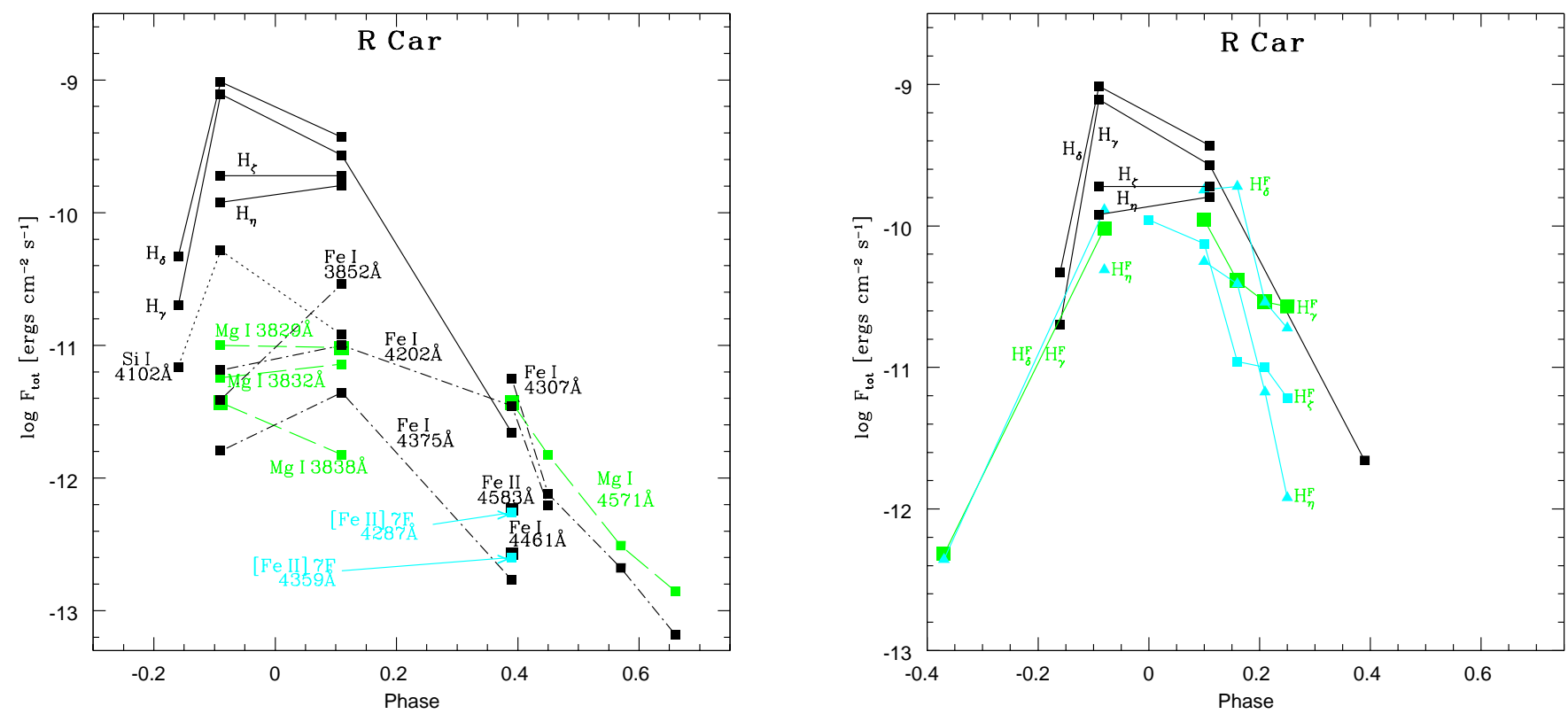

Fig. 22. Left: Absolute line fluxes plotted against phase in R Car. Note that there are coincident points for the phases $\phi=-0.09$ $(\mathrm{MgI} 3838 \AA$ and FeI $3852 \AA), \phi=0.11(\mathrm{MgI} 3829 \AA$ and FeI $4202 \AA)$ as well as at phase $\phi=0.39(\mathrm{FeI} 4202 \AA$ and $\mathrm{MgI}$ $4571 \AA$; Fe II $4583 \AA$ and [Fe II] $7 \mathrm{~F} 4287 \AA$; Fe I $4461 \AA$ and [Fe II] $7 \mathrm{~F} 4359 \AA$ ). Right: Balmer line fluxes compared to those of Fox et al. (1984), which are plotted in grey and marked with $\mathrm{H}_{\gamma}^{F}, \mathrm{H}_{\delta}^{F}, \mathrm{H}_{\zeta}^{F}$ and $\mathrm{H}_{\eta}^{F}$ (coincident fluxes: $\phi=-0.37 \mathrm{H}_{\gamma}^{F}$ and $\mathrm{H}_{\delta}^{F}$, $\phi=0.16 \mathrm{H}_{\gamma}^{F}$ and $\mathrm{H}_{\eta}^{F}$ and $\phi=0.21 \mathrm{H}_{\gamma}^{F}$ and $\mathrm{H}_{\delta}^{F}$ )

half a cycle after the shock emerges from the deep photosphere. Since the lines are still in emission, the shock must still be propagating outward so that the post-shock material, from which the line emission presumably originates, should show a positive outward velocity. (We note in pass- ing that since pulsation in the outer layers can be quite irregular (e.g. Bessell et al. 1996), in an individual cycle the shock could be stalled or even reversed by infalling material from a previous cycle, but, on average, shocks must progress outward.) By the time of minimum light, the 


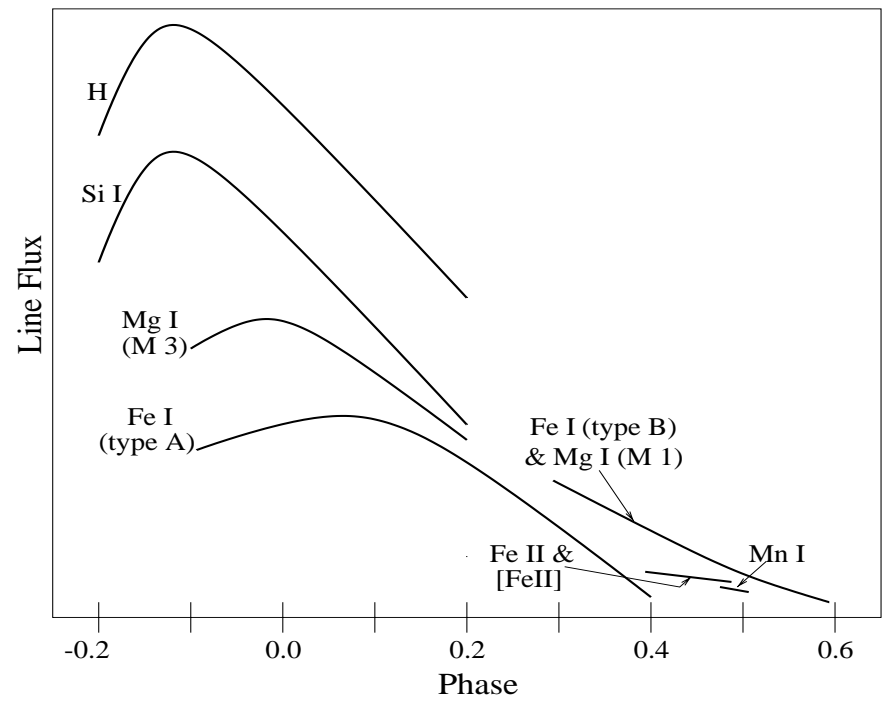

Fig. 23. Simplified time sequence for the observed emission lines and their total fluxes (in arbitary units)

shock is far above the photosphere (defined for the present discussion to be at optical depth one). For example, in a typical fundamental mode Mira model (e.g. see the models of Bessell et al. 1996 or Hofmann et al. 1998), the photosphere is at $\sim 240 R_{\odot}$ while the shock is at $\sim 420 R_{\odot}$. In a simple geometric model for emission from such a system, neglecting absorption above the photosphere, the emission lines would have square profiles going from $+v_{\text {shock }}$ to $-0.82 \times v_{\text {shock}}$, where $v_{\text {shock }}$ would be $\sim 5 \mathrm{~km} \mathrm{~s}^{-1}$ : only the emission with the most negative velocity is hidden behind the star. We would therefore expect the line emission to be centered close to velocity zero, as observed. Detailed models for the transfer of line photons originating from the shock are needed to make quantitative estimates of shock velocities high in the Mira atmosphere.

\section{Summary}

A sample of six M-type Miras has been observed with a phase coverage of about one pulsation period. We obtained time-resolved, high-dispersion emission line spectra and line fluxes in the optical spectral region for the Balmer lines $(\mathrm{H} \gamma, \mathrm{H} \delta, \mathrm{H} \zeta, \mathrm{H} \eta)$ and for metal lines of $\mathrm{Mg} \mathrm{I}, \mathrm{MnI}$, Si I, Fe I, Fe II and [FeII]. The variation of line shape, velocity and flux with phase has been discussed.

Evidence was presented that brighter maxima are associated with stronger shock waves. In R Car, the Balmer line fluxes we observed around maximum were much stronger than the fluxes observed by Fox et al. (1984), and the maximum during which we observed the Balmer lines was $\sim 1$ mag. brighter than the maximum during which Fox et al. observed. In R Leo, the second minimum during which we observed showed much stronger metal emission lines than the first, and the second minimum followed a brighter maximum than the first. Forbidden [Fe II] lines were only observed during the second minimum of R Leo and after the relatively bright maximum of $\mathrm{R}$ Car. This

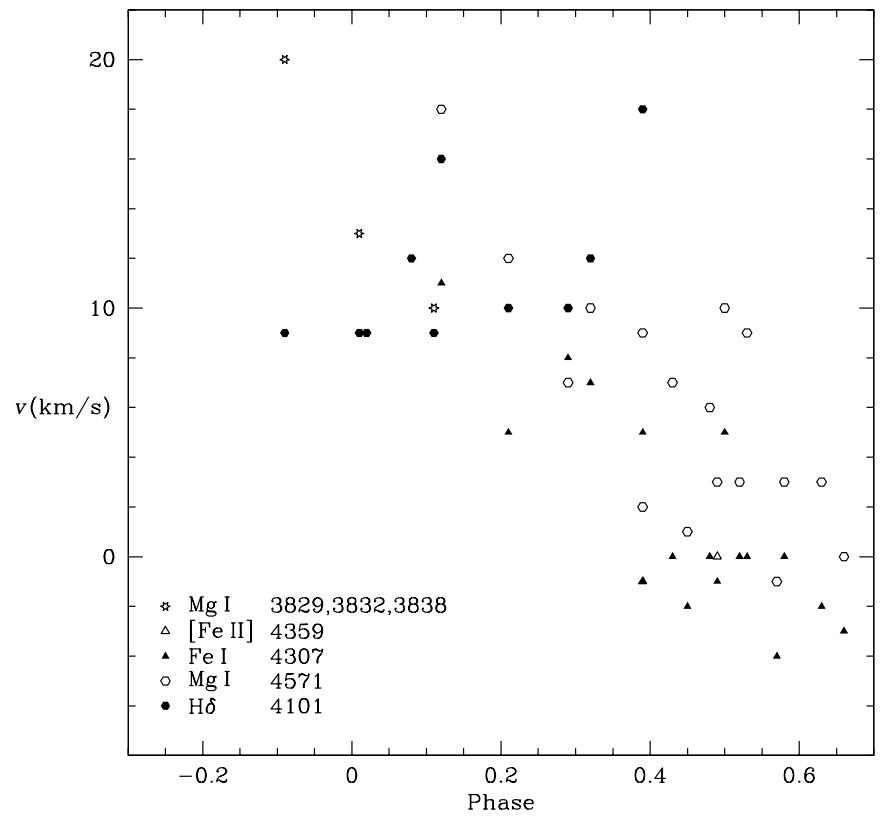

Fig. 24. The velocities of representative emission lines plotted against the phase of the pulsation cycle

suggests that their erratic appearance may be because they require an exceptionally bright maximum for production.

A proper interpretation of the observations presented in this paper will require the help of detailed theoretical modelling. Our future work will involve self-consistent shock models for the production of the observed emission lines and radiative transfer calculations in moving atmospheres under conditions of non-LTE, to model the transfer of the emission line photons through the Mira atmosphere.

Acknowledgements. This work was supported by the Deutscher Akademischer Austauschdienst, DAAD with a HSP III grant in 1999 , project number D/99/15656. In 2000 this work was supported by the Deutsche Forschungsgemeinschaft, DFG project number SE 420/21-1.

\section{References}

Aoki, W., Tsuji, T., \& Ohnaka, K. 1998, A\&A, 333, L19

Bessell, M. S., Scholz, M., \& Wood, P. R. 1996, A\&A, 307, 481

Bowen, G. H. 1988, ApJ, 329, 299

Cernicharo, J., Alcolea, J., Baudry, A., et al. 1997, A\&A, 319, 607

Deutsch, A. J., \& Merrill, P. W. 1959, ApJ, 130, 570

Feuchtinger, M. U., Dorfi, E. A., \& Höfner, S. 1993, A\&A, 273, 513

Fleischer, A. J., Gauger, A., \& Sedlmayr, E. 1992, A\&A, 266, 321

Fox, M. W., Wood, P. R., \& Dopita, M. A. 1984, ApJ, 286, 337

Fox, M. W., \& Wood, P. R. 1985, ApJ, 297, 455

Fuhr, J. R., Martin, G. A., \& Wiese, W. L. 1988, J. Phys. Chem. Ref. Data 17, Suppl. 4 
Gillet, D., \& Lafon, J. P. J. 1983, A\&A, 128, 53

Gillet, D., Bouchet, P., Ferlet, R., et al. 1985a, ESO Messenger, 3,38

Gillet, D., Maurice, E., Bouchet, P., et al. 1985c, A\&A, 148, 155

Gillet, D., Ferlet, R., Maurice, E., et al. 1985b, A\&A, 150, 89

Gorbatskii, V. G. 1961, SvA, 5, 192

Groenewegen, M. A. T., Baas, F., Blommaert, J. A. D. L., et al. 1999, A\&AS, 140, 197

Habing, H. 1996, A\&AR, 7, 97

Hinkle, K. H. 1978, ApJ, 220, 210

Hinkle, K. H., Hall, D. N. B., \& Ridgway, S. T. 1982, ApJ, 252, 697

Hinkle, K. H., Scharlach, W. W. G., \& Hall, D. N. B. 1984, ApJS, 56, 1

Hofmann, K.-H., Scholz, M., \& Wood, P. R. 1998, A\&A, 339, 846

Höfner, S., \& Dorfi, E. 1997, A\&A, 319, 648

Joy, A. H. 1947, ApJ, 106, 288

Joy, A. H. 1954, ApJS, 1, 39

Mattei, J. A., Mayer, E. H., \& Baldwin, M. E. 1980, Sky Telesc. 60,180
Merrill, P. W. 1940, Spectra Of Long-Period Variable Stars (University of Chicago Press)

Merrill, P. W. 1945, ApJ, 102, 347

Merrill, P. W. 1946a, PASP, 58, 304

Merrill, P. W. 1946b, ApJ, 103, 275

Merrill, P. W. 1947a, ApJ, 105, 360

Merrill, P. W. 1947b, ApJ, 106, 274

Nogami, D., Kato, T., Baba, H., et al. 1997, Electronic Publishing, Now and the Future, Joint Discussion 12, 23rd meeting of the IAU, Kyoto, Japan

Reid, M. J., \& Dickson, D. F. 1976, ApJ, 209, 505

Thackeray, A. D. 1937, ApJ, 86, 499

Willson, L. A. 1976, ApJ, 205, 172

Winters, J. M., Le Bertre, T., Jeong, K. S., et al. , A\&A, 361, 641

Wood, B. E., \& Karvovska, M. 2000, ApJ, 535, 304

Wood, P. R. 1979, ApJ, 227, 220

Wood, P. R. 1987, in Stellar Pulsation, ed. A. N. Cox, W. M. Sparks \& S. G. Starrfield Lecture Notes (Springer-Verlag: Berlin), in Physics vol. 274, 250

Young, K. 1995, ApJ, 445, 872 